\title{
Effect of Cationic Surfactants on Properties of Zinc Oxide Nanoparticles Synthesized through Sol-gel Technique
}

\author{
Hwda Ghafur Rauf', Hamid Rashidi Nodeh², Madzlan Aziz², Sattar Ibrahim Kareem ${ }^{1}$ \\ ${ }^{1}$ Department of Medical Laboratory Sciences, University of Human Development, Sulaymaniyah, Kurdistan Region of \\ Iraq, ${ }^{2}$ Department of Science, University of Tehran, Tehran, ${ }^{3}$ Department of Science, University Technology Malaysia, \\ Johor Bahru, Malaysia
}

\section{A B S T R A C T}

Zinc oxide $(\mathrm{ZnO})$ is an important metal oxide which participates in lots of applications, including gas sensors, catalysis, and optoelectronic devices. For nanostructured materials, agglomeration is problematic in the chemical production processes. In surfactant-free chemical reactions, aggregation occurs immediately as particles are generated. Therefore, the synthesis of controllable size nanoparticles is of great fundamental and technological interest. In the present study, the effect of cationic surfactants such as cetyltrimethylammonium bromide and benzalkonium chloride concentrations, on the optical properties, size, and morphology of ZnO nanoparticles synthesized through sol-gel method were studied. The characterizing tools were X-ray diffraction, Fourier transform infrared (FTIR) spectroscopy, scanning electron microscopy (SEM), transmission electron microscopy (TEM), and ultraviolet-visible (UV-Vis-near-infrared) spectrophotometer. X-ray diffractogram confirmed the hexagonal (wurtzite structure) of $\mathrm{ZnO}$. FTIR analyzed the presence of functional groups. The shape and size of the $\mathrm{ZnO}$ nanostructures were revealed by SEM and TEM. The band gap energy of the prepared ZnO samples was determined by UV-Vis spectrophotometer.

Index Terms: Benzalkonium Chloride, Cationic Surfactants, Cetyltrimethyl Ammonium Bromide, Nanostructures, Sol-gel Method, Zinc Oxide

\section{INTRODUCTION}

Zinc oxide $(\mathrm{ZnO})$ is an interesting material as it is a semiconductor that has a large exciton binding energy of 60 $\mathrm{MeV}$ with a wide band gap of $3.4 \mathrm{eV}$ at room temperature. The study of synthesis and growth of $\mathrm{ZnO}$ due to its sizedependent optical absorption is always a valuable tool. In fact, in an accessible size range experimentally, $\mathrm{ZnO}$ can show

\begin{tabular}{|l|l|}
\hline \multicolumn{2}{|c|}{ Access this article online } \\
$\begin{array}{l}\text { DOI:10.21928/undjst.v3n2y2019.pp93-107 } \\
\text { E-ISSN: 2521-4217 } \\
\text { P-ISSN: 2521-4209 }\end{array}$ \\
\hline $\begin{array}{l}\text { Copyright @ } 2019 \text { Al-Janabi, et al. This is an open access article } \\
\text { distributed under the Creative Commons Attribution Non-Commercial } \\
\text { No Derivatives License 4.0 (CC BY-NC-ND 4.0) }\end{array}$ \\
\hline
\end{tabular}

quantum confinement effects, unlike many oxides. Therefore, to quantum size $\mathrm{ZnO}$ particles, the best method to be used in cases like this is the sol-gel preparation method [1]. Not to mention $\mathrm{ZnO}$ has a low-temperature process in addition to cost savings, high carrier mobility, and transparency [1]. In the production and use of many chemical and pharmaceutical products, the most problematic matter is agglomeration. This aggregation immediately occurs when particles are generated in surfactantfree chemical reactions. To prevent aggregation, we have to embark from conventional colloid science in which particles are coated with foreign capping agents and/or the surface charges are tailored to separate them through electrostatic repulsions [2]. Because of its great fundamental and technological interest, it is always favorable to synthesize the ultrafine and controllable size particles with nanocrystalline structures [3].

\footnotetext{
Corresponding author's e-mail: Hwda Ghafur Rauf, University of Human Development, Sulaymaniyah, Kurdistan Region, Iraq.
} E-mail: hwda.rauf@uhd.edu.iq 
Most surfactants have a hydrophilic (water-loving) head and a long hydrophobic (water-hating or oil-loving) tail, as shown in Figs. 1 and 2. We often describe surfactants is being amphiphilic molecules - they love everything [4]. Due to their capability to control crystal growth and to provide solubility, surfactants are widely used during the preparation of crystalline metal oxide in the nanoscale, which by organizing the small oxide crystallites into a controlled structure, they can maintain regular pores and low angle Bragg reflection [5]. Previous studies showed that cationic surfactants generally affect the morphology, size, and optical properties of metal oxides by controlling the particle's growth. Cetyltrimethylammonium bromide (CTAB) is a common surfactant used in nanoparticle synthesis. One of CTAB's uses is to direct the growth and stabilize the shape of nanoparticles [6]. Although the study on benzalkonium chloride (BAK) effect on metal oxide properties is still unknown but it has similar uses to other cationic surfactants [7]. However, the variety in the molecular structure of the surfactants was taken into consideration to choose BAK that consists of a head group and a double ring bi-tailed structure.

Several physical and chemical methods could be taken under consideration to synthesize $\mathrm{ZnO}$ nanostructures. Chemical vapor deposition (CVD), metal-organic CVD, and molecular beam epitaxy, pyrolysis, vapor-liquid-solid growth, and vaporsolid processes such as thermal reduction, are considered physical methods. At the same time, to have a very large scale production, there are chemical methods that are very simple yet effective such as precipitation, sol-gel, and solvothermal processes. Unlike general methods, these methods do not require high temperatures and sophisticated instruments [8]. In this work, the purpose is to synthesize a controllable grain size of particles, to approach that goal, the sol-gel method is the most convenient process, especially when we adjust these experimental conditions such as concentration, temperature, $\mathrm{pH}$, and reaction time. Besides, its simplicity, this method is reproducible and cost affective, as well as its the reliability of stoichiometry control, which makes it a thoroughly suitable process for industrial production of $\mathrm{ZnO}$ [9].

\section{EXPERIMENTAL}

\subsection{Apparatus}

The tools that have been used in this experiment are silica crucible, measuring cylinder, electronic balance, filter tunnel, spatula, plastic wash bottle, beaker, Petri dish, desiccators, beaker $(100-500 \mathrm{~mL})$, volumetric flask (100-250 mL), plastic tubes, magnetic stir bar, hotplates, filter paper, forceps, gloves, containers, Pyrex glasses substrate, furnace, oven, and the centrifuge instrument.

\subsection{Preparation of $\mathrm{ZnO}$ Nanoparticles without and with (CTAB) and (BAK) Individually}

$\mathrm{ZnO}$ nanocrystals were prepared by adding $100.0 \mathrm{~mL}$ of $0.5 \mathrm{~mol} / 1 \mathrm{NaOH}$ solution dropwise slowly into $250.0 \mathrm{~mL}$ of $0.1 \mathrm{~mol} / 1 \mathrm{Zn}(\mathrm{Ac})_{2}$ solution under vigorous stirring and closed vessel to produce the $\mathrm{Zn}(\mathrm{OH})_{2}$ precipitate, then an appropriate amount of $\mathrm{NH}_{4} \mathrm{HCO}_{3}(0.8 \mathrm{~g})$ powder was added. After stirring for $30 \mathrm{~min}$, a semitransparent zinc carbonate hydroxide colloid was obtained. After $30 \mathrm{~min}$, the colloid was centrifuged and

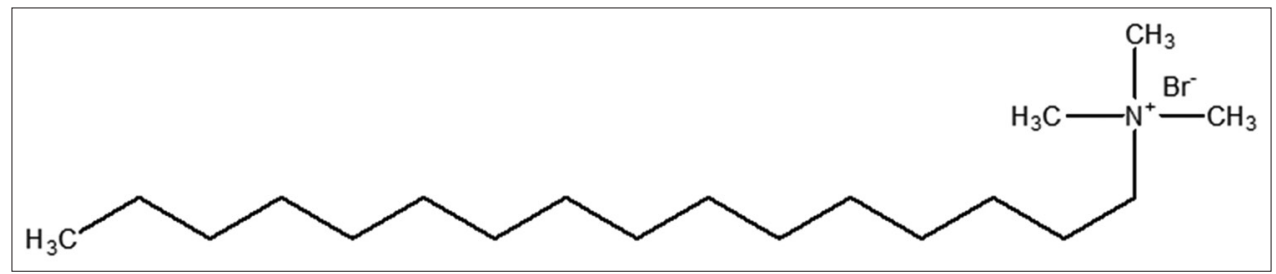

Fig. 1. Chemical structure of cetyltrimethylammonium bromide surfactant $\mathrm{CH}_{3}\left(\mathrm{CH}_{2}\right)_{15} \mathrm{~N}\left(\mathrm{CH}_{3}\right) 3+\mathrm{Br}^{-}$[4].

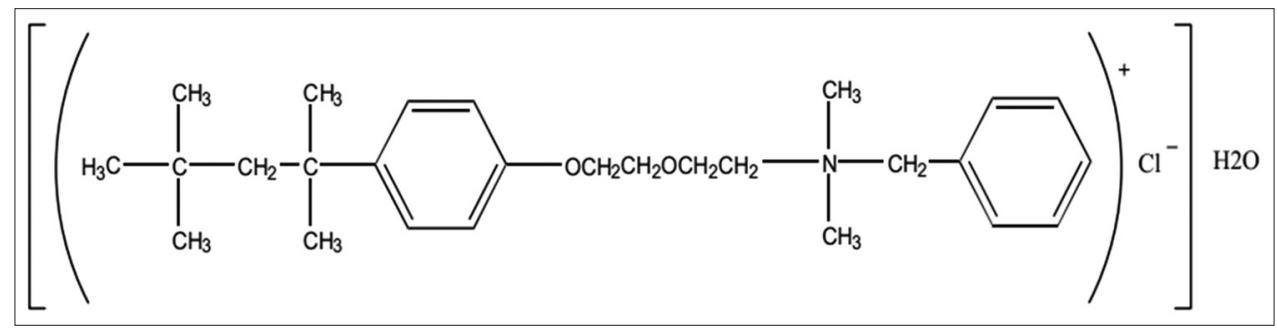

Fig. 2. Structure of benzalkonium chloride [7]. 
dried at $80^{\circ} \mathrm{C}$. Thus, the precursor of a small crystallite of $\mathrm{Zn}_{5}\left(\mathrm{CO}_{3}\right)_{2}(\mathrm{OH})_{6}$ with white color was formed then calcinated at $450^{\circ} \mathrm{C}$ for $2 \mathrm{~h}$. Finally, the product was dried at $70^{\circ} \mathrm{C}$ to obtain the white-colored sample of $\mathrm{ZnO}$ nanoparticle.

In the case of $\mathrm{CTAB}$, after adding $\mathrm{NH}_{4} \mathrm{HCO}_{3}$ powder then CTAB $\left(5 \times 10^{-4} \mathrm{M}\right.$ below, $9 \times 10^{-4} \mathrm{M}$ at, $15 \times 10^{-4} \mathrm{M}$ above $)$ critical micelle concentration (CMC) was added individually. After stirring for $30 \mathrm{~min}$, a semitransparent zinc carbonate hydroxide, CTAB colloid was obtained. After $30 \mathrm{~min}$, the colloid was centrifuged and dried at $80^{\circ} \mathrm{C}$. Thus, the precursor of a small crystallite of $\mathrm{Zn}_{5}\left(\mathrm{CO}_{3}\right)_{2}(\mathrm{OH})_{6}$ : CTAB with white color was formed. The precursor was then calcinated at $450^{\circ} \mathrm{C}$ for $2 \mathrm{~h}$ to obtain the sample. Finally, the product was dried at $70^{\circ} \mathrm{C}$ to obtain the white-colored sample of $\mathrm{ZnO}$.

On the other hand, this time instead of CTAB, BAK $\left(1 \times 10^{-5} \mathrm{M}\right.$ below, $5 \times 10^{-5} \mathrm{M}$ at, $15 \times 10^{-5} \mathrm{M}$ above $)$, and $\mathrm{CMC}$ were added one at a time. After stirring for $30 \mathrm{~min}$, a semitransparent zinc carbonate hydroxide, BAK colloid was obtained. After $30 \mathrm{~min}$, the colloid was centrifuged and dried at $80^{\circ} \mathrm{C}$. Thus, the precursor of a small crystallite of $\mathrm{Zn}_{5}\left(\mathrm{CO}_{3}\right)_{2}(\mathrm{OH})_{6}$ : BAK with white color was formed. The precursor was then calcinated at $550^{\circ} \mathrm{C}$ for $5 \mathrm{~h}$ to obtain the sample. Finally, the product was dried at $70^{\circ} \mathrm{C}$ to obtain the white-colored sample of $\mathrm{ZnO}$ [10].

\subsection{Determination of CMC of CTAB Surfactant}

$\mathrm{CMC}$ value determination of the CTAB surfactant was done using the conductivity method. Experiment was taken place at $298 \mathrm{~K}$ using thermostated water bath [10]-[13]. A CMC of the surfactant solutions was prepared using distilled water. These solutions were left for 15-20 min before being added to the $\mathrm{ZnO}$ synthesis precursor solution and measurements. The electrical conductivity was measured using conductivity meter, model ECCON1103K made from Singapore.

\section{RESULTS AND DISCUSSION}

\subsection{CMC of CTAB}

Fig. 3 shows that the CMC of CTAB was determined by plotting conductivity values against the concentration of surfactant solutions. The CMC value is obtained from the interception of two conductivity lines [11], the result was found to be $0.9 \mathrm{mM}$ this result is near to that given in the literature data $0.89 \mathrm{mM}$ [12]. The difference might be caused by taking the reading of conductivity meter before the conductivity meter could display a constant value. The CMC value of $\mathrm{BAK}$ was taken from literature data and it was $0.04 \mathrm{mM}$ [13].

\subsection{Analysis of Fourier Transformed Infrared (FTIR)}

Fig. 4a shows the FTIR spectrum of prepared $\mathrm{ZnO}$ nanoparticles without CTAB. Furthermore, Fig. 4b-d shows the FTIR spectra of prepared $\mathrm{ZnO}$ nanoparticles in the presence of CTAB (below CMC, at CMC, and after CMC), respectively, while Fig. 5 shows the FTIR spectra of prepared $\mathrm{ZnO}$ nanoparticles in the presence of BAK (below CMC, at $\mathrm{CMC}$, and after $\mathrm{CMC}$ ), respectively. The broad absorption band centered above $3000 \mathrm{~cm}^{-1}$ is attributable to the band $\mathrm{O}-\mathrm{H}$ stretching vibrations of water molecules on $\mathrm{ZnO}$, and the band near $1630 \mathrm{~cm}^{-1}$ is assigned to the bond in $\mathrm{H}-\mathrm{O}-\mathrm{H}$ bending vibrations mode were also presented due to the adsorption of humidity in the air when FT-IR sample disks were prepared in an open air.

The spectrums near $1450 \mathrm{~cm}^{-1}$ indicate the existence of $\mathrm{C}-\mathrm{O}$. The broad absorption band in the region $1030-1384 \mathrm{~cm}^{-1}$ is assigned to $\mathrm{C}-\mathrm{H}$ long-chain methyl, probably this is due to impurities like CTAB remnants. The existence of extra spectrums in Fig. 4b-d could indicate the unsuccessful removal of surfactant due to the calcination low temperature, which was $450^{\circ} \mathrm{C}$ and for $2 \mathrm{~h}$. However, for $\mathrm{ZnO}$ samples with $\mathrm{BAK}$ surfactant, the calcination temperature was raised to $550^{\circ} \mathrm{C}$ for $5 \mathrm{~h}$. The difference in the calcination temperatures and the time could explain the difference between the figures, and because of using two different surfactants, Fig. 4b-d of $\mathrm{ZnO}$ samples with CTAB surfactant is generally blue-shifted which means a decrease in wavelength [14] while Fig. 5 of $\mathrm{ZnO}$ samples with BAK surfactant is not blue shifted.

\subsection{Analysis of Scanning Electron Microscopy (SEM)}

The morphology of the prepared $\mathrm{ZnO}$ samples was determined by SEM analysis, as shown in Figs. 6-12,

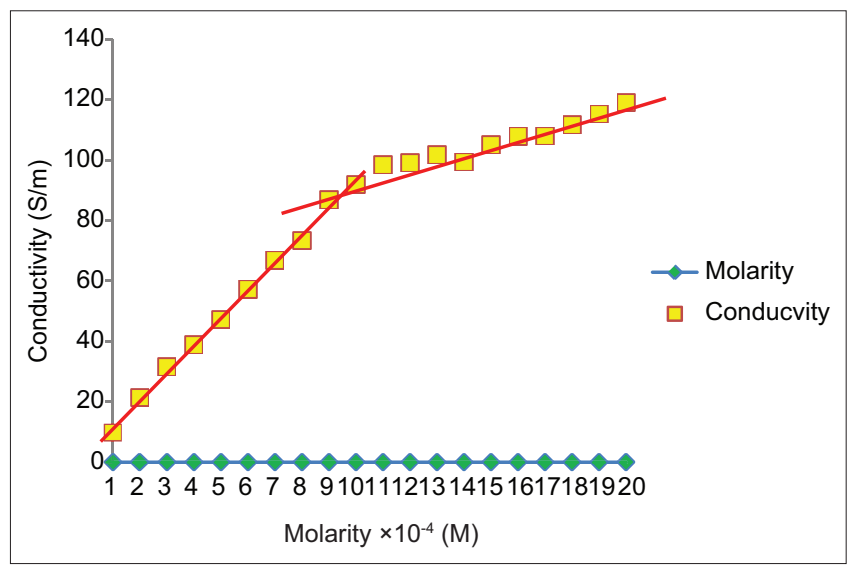

Fig. 3. Plot of conductivity against cetyltrimethylammonium bromide concentration, the temperature is at $298 \mathrm{~K}$. 


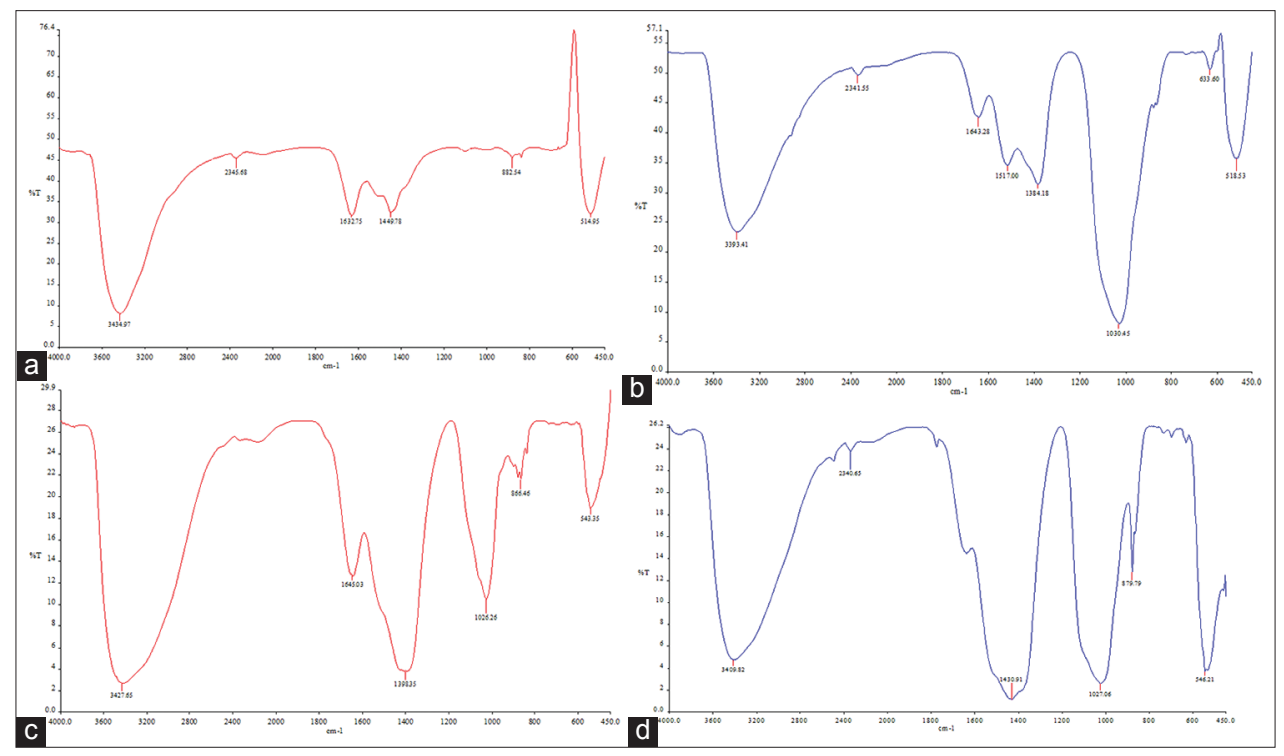

Fig. 4. Combined Fourier transform infrared spectrums of zinc oxide nanoparticles without and with cetyltrimethylammonium bromide (CTAB) surfactant. (a). Without surfactant, (b) with CTAB below critical micelle concentration (CMC), (c) with CTAB at CMC, (d) with CTAB above CMC.

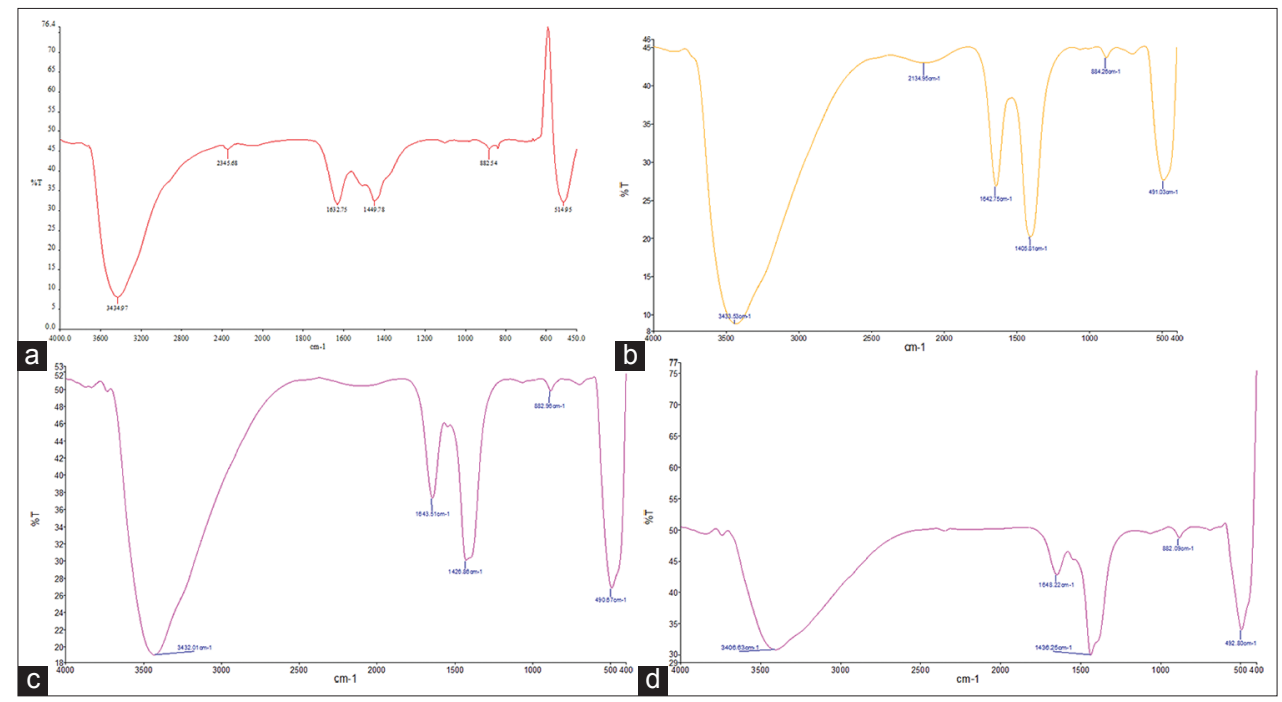

Fig. 5. Combined Fourier transform infrared spectra of zinc oxide nanoparticles without and with benzalkonium chloride (BAK) surfactant. (a). Without surfactant, (b) with BAK below critical micelle concentration (CMC), (c) with BAK at CMC, (d) with BAK above CMC.

$\mathrm{ZnO}$ samples with $\mathrm{CTAB}$ are calcined at $450^{\circ} \mathrm{C}$ for $2 \mathrm{~h}$; demonstrate uniform but not the best morphology, due to impurities that have been investigated by FTIR results. $\mathrm{ZnO}$ samples with $\mathrm{BAK}$ are calcined at $550^{\circ} \mathrm{C}$ for $5 \mathrm{~h}$, do not reveal uniform morphology. The structure of BAK is bulky and bi-tailed which is insoluble; the particles tend to agglomerate, which caused the increase in the particle size [15]. The particle sizes of $\mathrm{ZnO}$ sample were taken by line intersecting method; the result revealed that the prepared $\mathrm{ZnO}$ sample without CTAB and BAK in Fig. 4 was in the range of $80-85 \mathrm{~nm}$ which was larger than the prepared samples in the presence of CTAB (Figs. 7-9) and smaller than the prepared samples in the presence of BAK (Figs. 10-12), respectively. Among the other samples, the particle size of $\mathrm{ZnO}$ with $\mathrm{CTAB}$ at $\mathrm{CMC}$, as shown in Fig. 8, was in the range of $55-60 \mathrm{~nm}$. Both surfactants have a long chain of carbon included compound, in CTAB carbon may surround the zinc $(\mathrm{Zn})$ particle or located between them, resulting in reduction of particle size and minimize the agglomeration of particles.

Although SEM is not an accurate technique to determine the particle size, using line intersecting method according 


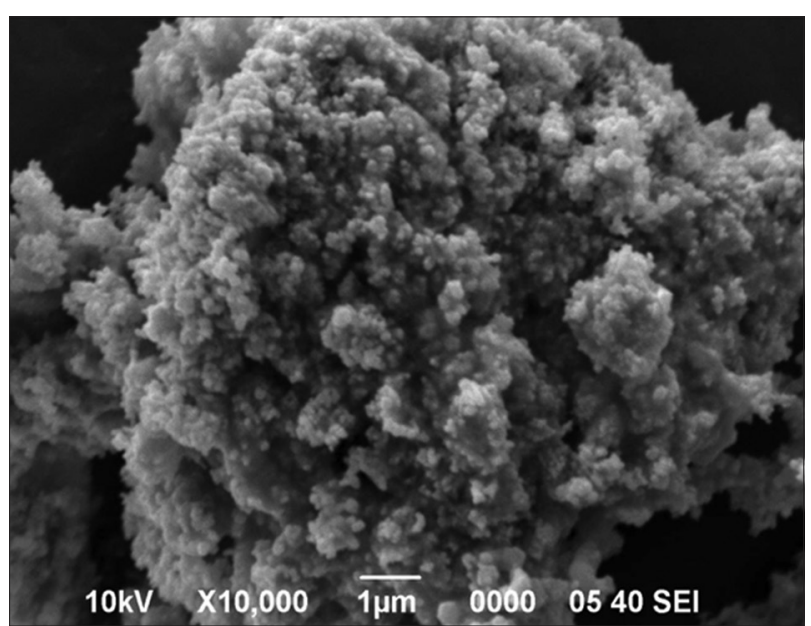

Fig. 6. Scanning electron microscopy image of zinc oxide nanoparticles without surfactant.

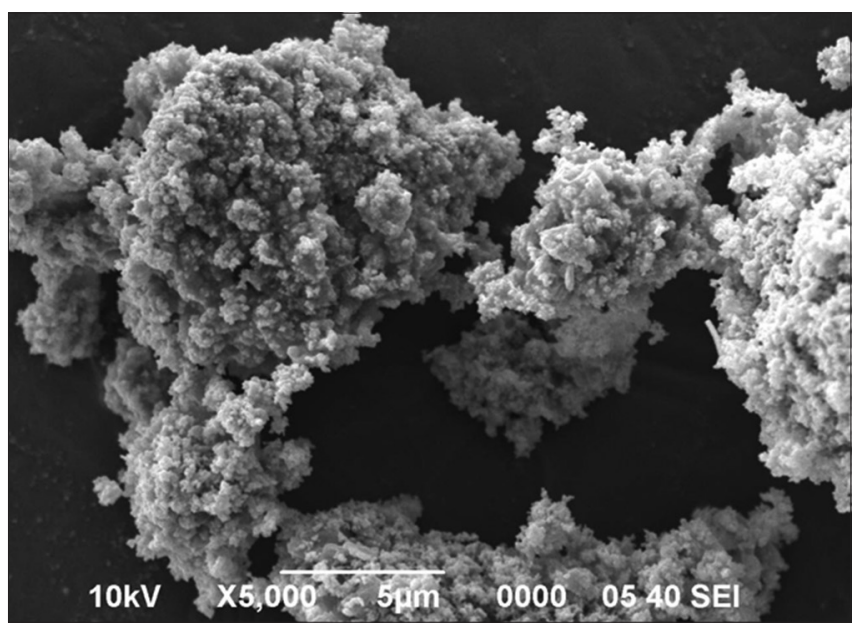

Fig. 7. Scanning electron microscopy image of zinc oxide nanoparticles with cetyltrimethylammonium bromide below critical micelle concentration.

to particle size distributions of $\mathrm{ZnO}$ nanoparticles based on SEM images, $\mathrm{ZnO}$ particles without surfactant reveal an average size of $86 \mathrm{~nm}$, as shown in Fig. 13. The average size of samples with CTAB below CMC point is $84 \mathrm{~nm}$. From both figures, it can be concluded that there is not a considerable change. Nevertheless, $\mathrm{ZnO}$ samples at CMC point demonstrate a smaller average size of $73 \mathrm{~nm}$. The average size of samples with $\mathrm{CTAB}$ above $\mathrm{CMC}$ point is $80 \mathrm{~nm}$, as shown in Figs. 14-16.

However, the results for $\mathrm{ZnO}$ samples with $\mathrm{BAK}$ revealed a bigger average size of $125 \mathrm{~nm}$ for $\mathrm{ZnO}$ with $\mathrm{BAK}$ below CMC point, $145 \mathrm{~nm}$ for $\mathrm{ZnO}$ with $\mathrm{BAK}$ at $\mathrm{CMC}$ point, and $212 \mathrm{~nm}$ for $\mathrm{ZnO}$ with BAK above CMC point. These samples are not nanosized, as shown in Figs.

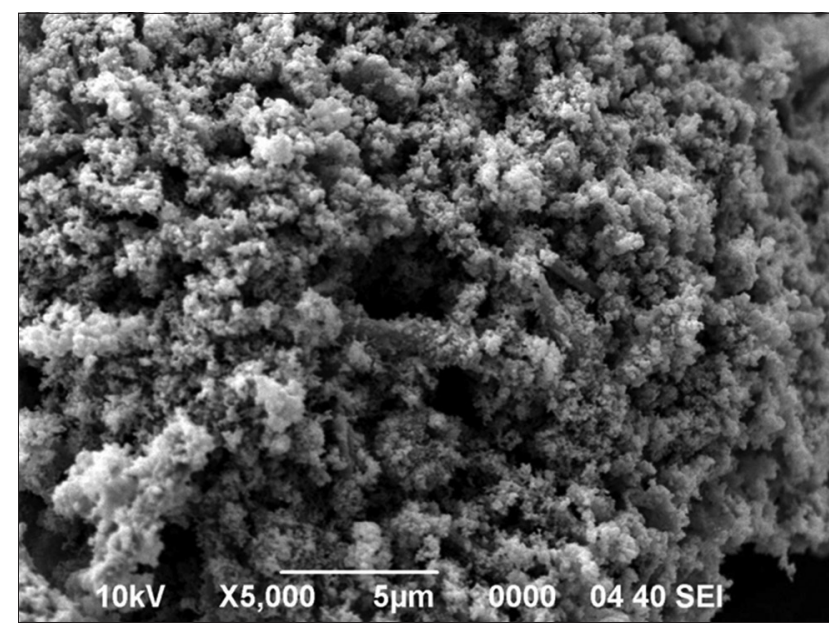

Fig. 8. Scanning electron microscopy image of zinc oxide nanoparticles with cetyltrimethylammonium bromide at critical micelle concentration.

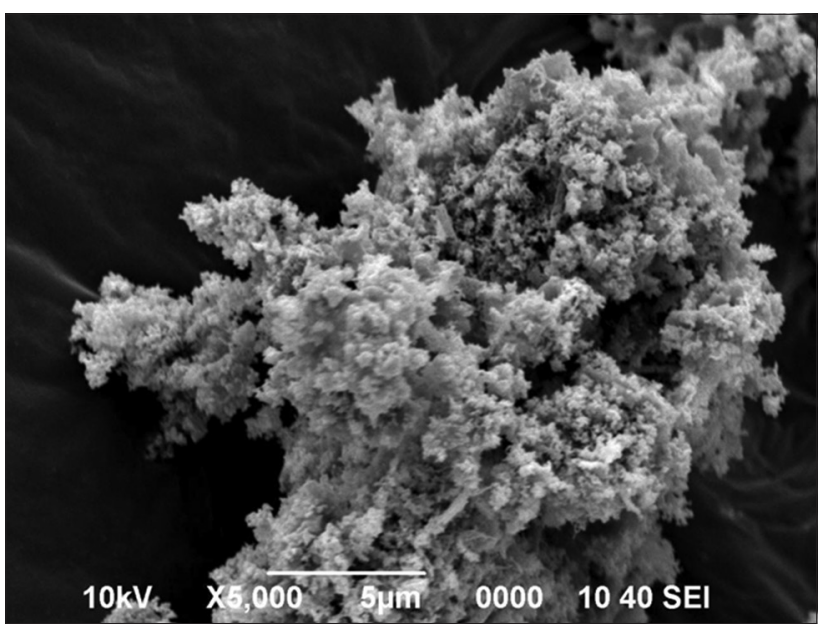

Fig. 9. Scanning electron microscopy image of zinc oxide nanoparticles with cetyltrimethylammonium bromide above critical micelle concentration.

17-19. The actual reason is due to BAK's bulky structure which contains 25 carbon atoms in the tail that cause the agglomeration, as a result of this led to an increase in the size of the particles [15].

\subsection{Analysis of Ultraviolet-Visible-Near-Infrared Spectroscopy}

Figs. 20-26 illustrates the $(\alpha h v)^{2}$ versus hu plot used for the estimation of the band gap of $\mathrm{ZnO}$ nanoparticles calcined at $550^{\circ} \mathrm{C}$ by extrapolating the graph to $\mathrm{X}$-axis so as to calculate the band gap of the samples. The band gap is found to be $3.26 \mathrm{eV}, 3.31 \mathrm{eV}, 3.49 \mathrm{eV}, 3.39 \mathrm{eV}, 3.27$ $\mathrm{eV}, 3.24 \mathrm{eV}$, and $3.21 \mathrm{eV}$ for the samples prepared without surfactant and with both surfactants CTAB and BAK 


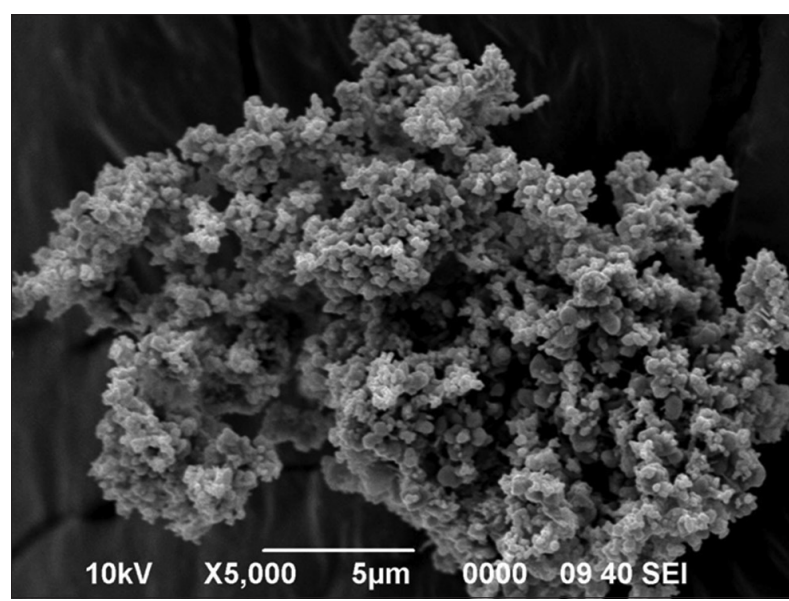

Fig. 10. Scanning electron microscopy image of zinc oxide nanoparticles with benzalkonium chloride below critical micelle concentration.

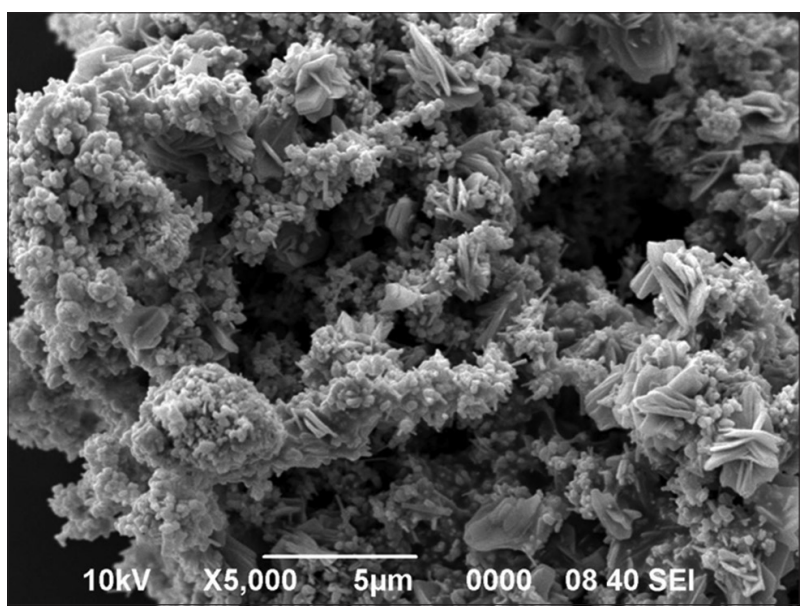

Fig. 11. Scanning electron microscopy image of zinc oxide nanoparticles with benzalkonium chloride at critical micelle concentration.

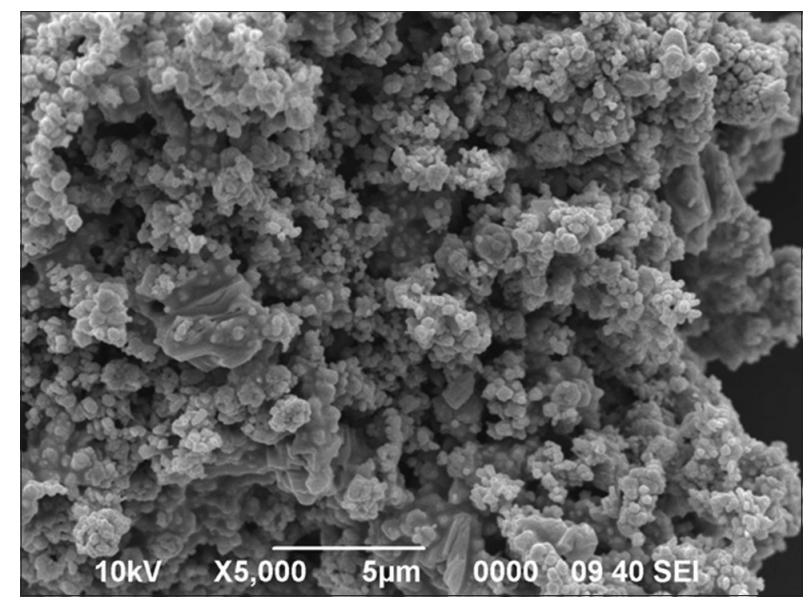

Fig. 12. Scanning electron microscopy image of zinc oxide nanoparticles with benzalkonium chloride above critical micelle concentration.

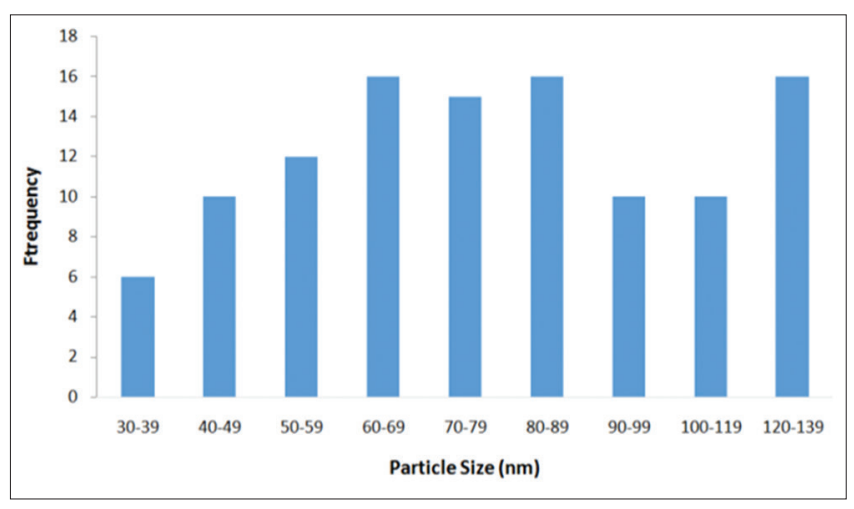

Fig. 13. Particle size distributions of zinc oxide nanoparticles without surfactant.

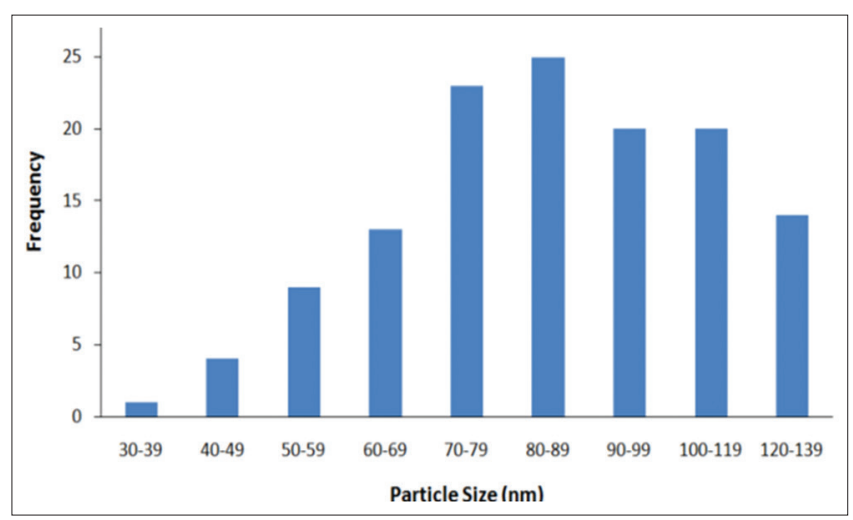

Fig. 14. Particle size distributions of zinc oxide nanoparticles with cetyltrimethylammonium bromide below critical micelle concentration.

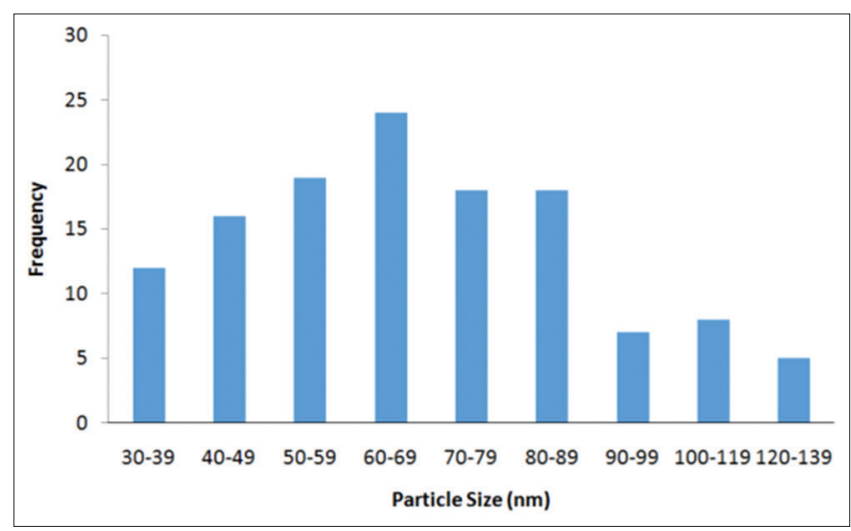

Fig. 15. Particle size distributions of zinc oxide nanoparticles with cetyltrimethylammonium bromide at critical micelle concentration.

individually at different concentration (below, at, and after), respectively. The band gap increases in the presence of CTAB particularly at CMC point due to quantum size effects, as the size of a particle decrease, till it reaches a nanoscale, the decrease in confining dimension makes the energy levels discrete and this increases or widens

UHD Journal of Science and Technology | Jul 2019 | Vol 3 | Issue 2 


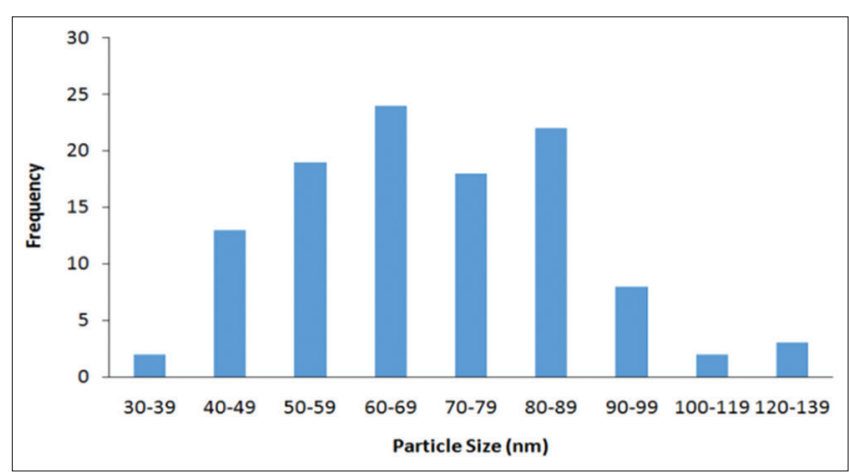

Fig. 16. Particle size distributions of zinc oxide nanoparticles with cetyltrimethylammonium bromide above critical micelle concentration.

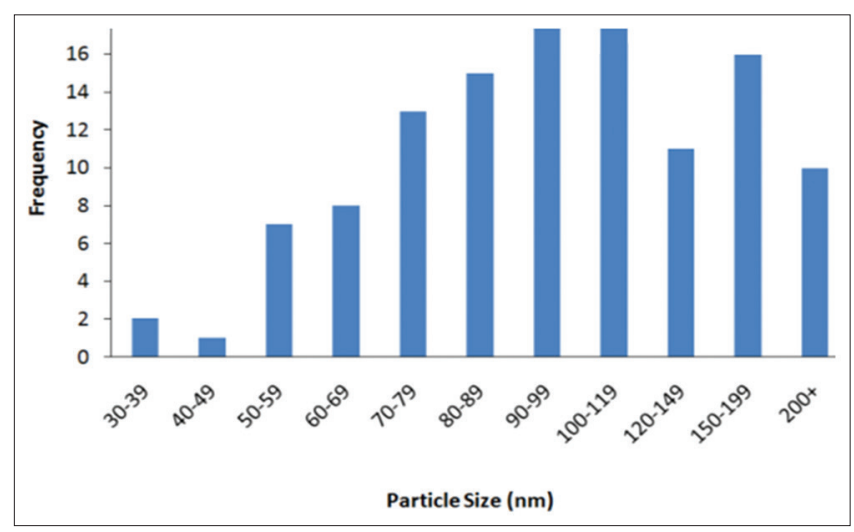

Fig. 17. Particle size distributions of zinc oxide nanoparticles with benzalkonium chloride below critical micelle concentration.

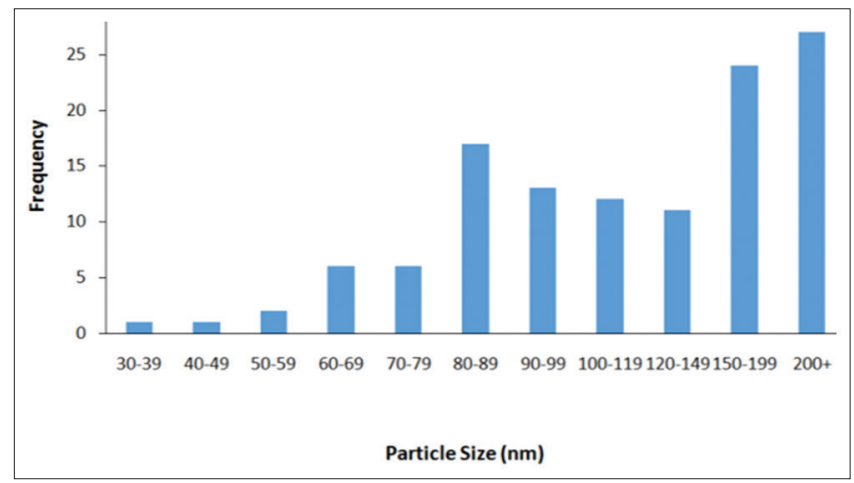

Fig. 18. Particle size distributions of zinc oxide nanoparticles with benzalkonium chloride at critical micelle concentration.

up the band gap and ultimately the band gap energy also increases [16], and decreases in the presence of BAK surfactant because of its bulky structure which contains 25 carbon atoms in the tails that cause the agglomeration. Although the results show two curves, which means impurities presence.

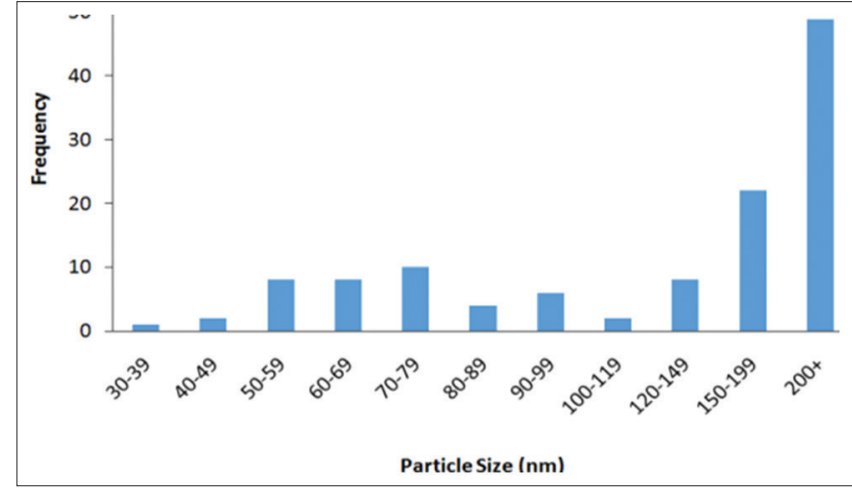

Fig. 19. Particle size distributions of zinc oxide nanoparticles with benzalkonium chloride above critical micelle concentration.

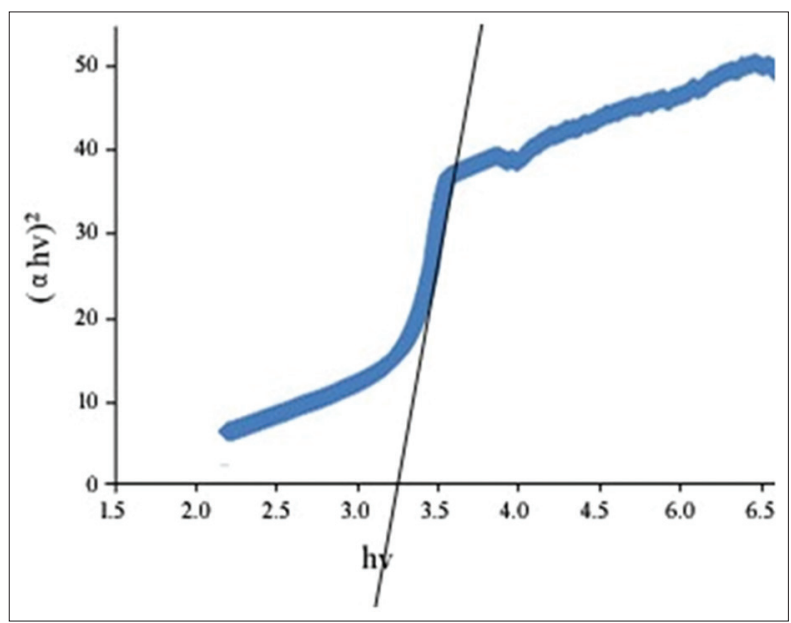

Fig. 20. Band gap energy for zinc oxide nanoparticles without surfactant.

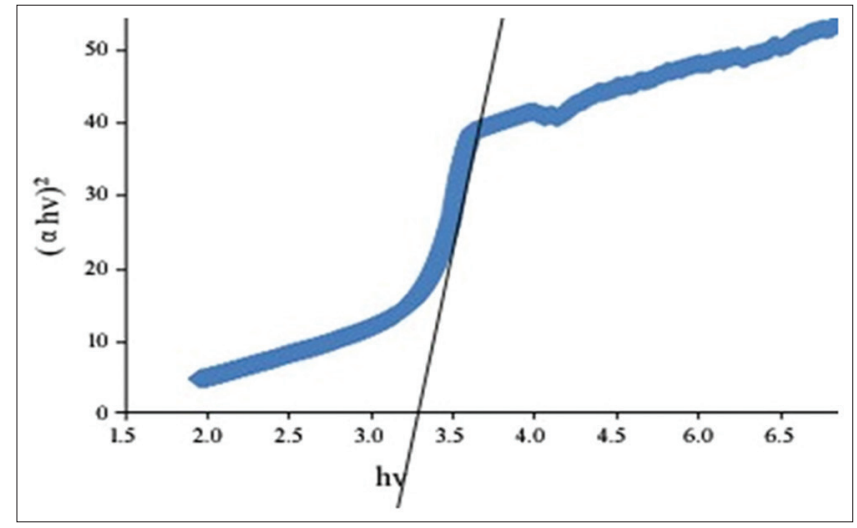

Fig. 21. Band gap energy for zinc oxide nanoparticles with cetyltrimethylammonium bromide below critical micelle concentration.

The following table (Table 1) reveals a comparison of band gap energy for each sample: 


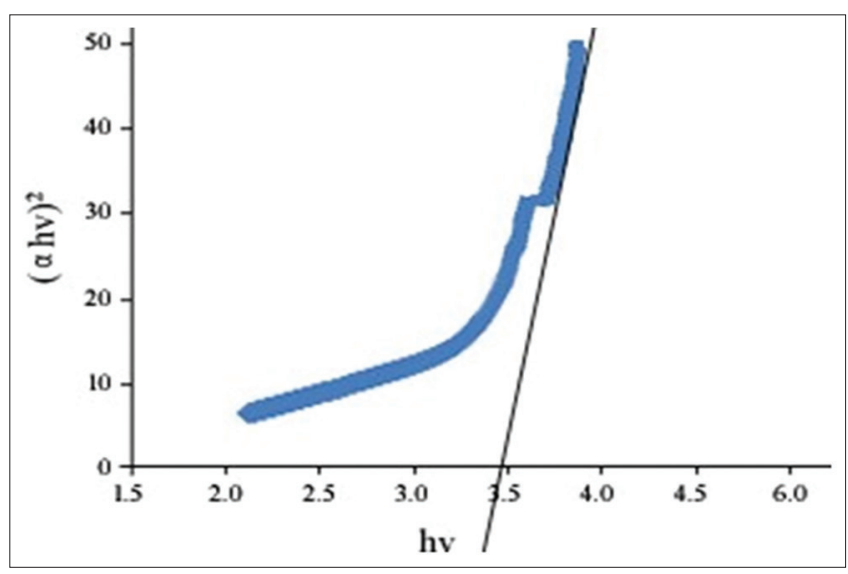

Fig. 22. Band gap energy for zinc oxide nanoparticles with cetyltrimethylammonium bromide at critical micelle concentration.

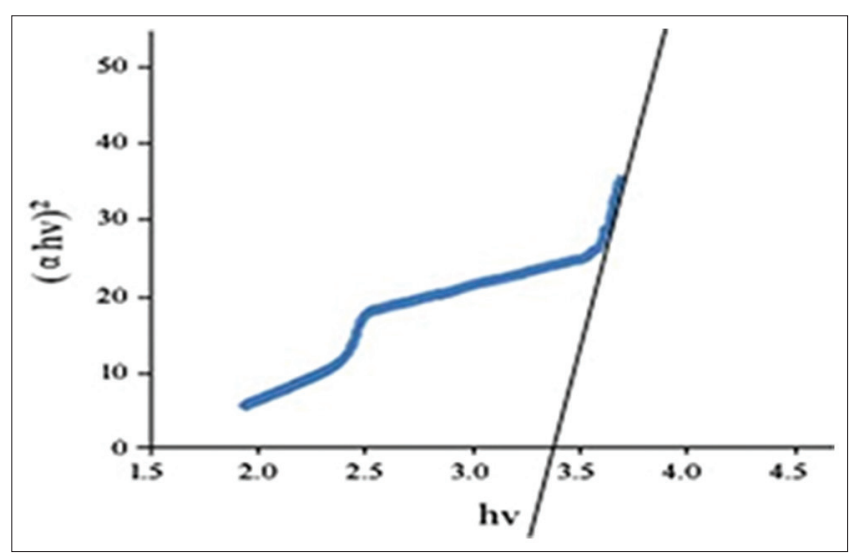

Fig. 23. Band gap energy for zinc oxide nanoparticles with cetyltrimethylammonium bromide above critical micelle concentration.

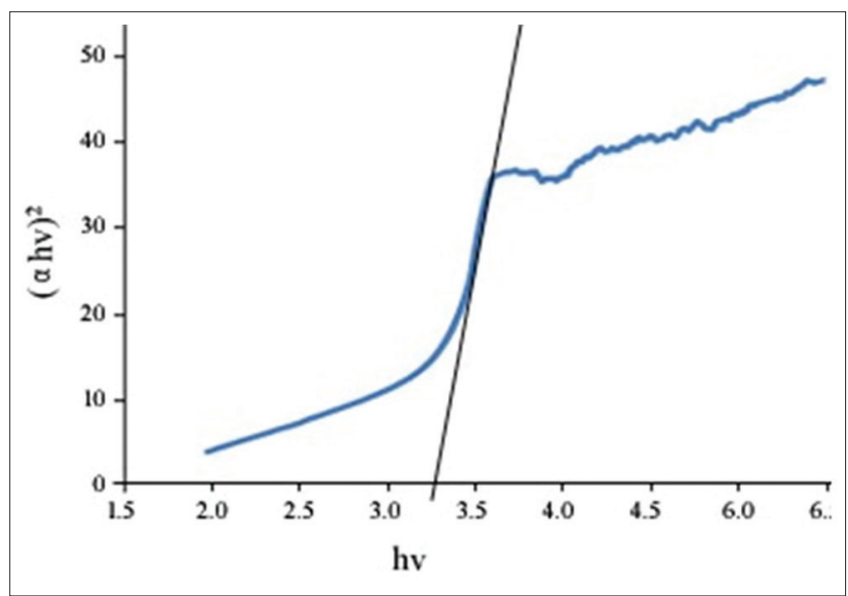

Fig. 24. Band gap energy for zinc oxide nanoparticles with benzalkonium chloride below critical micelle concentration.

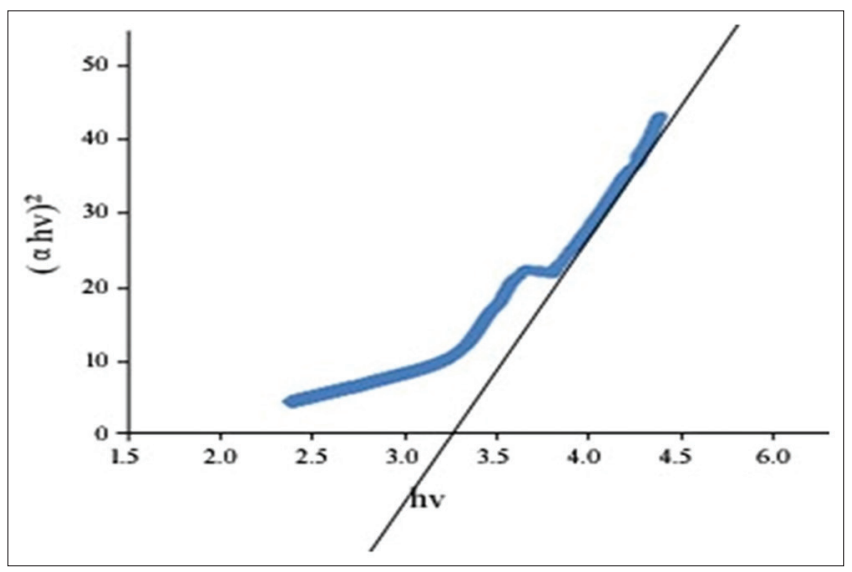

Fig. 25. Band gap energy for zinc oxide nanoparticles with benzalkonium chloride at critical micelle concentration.

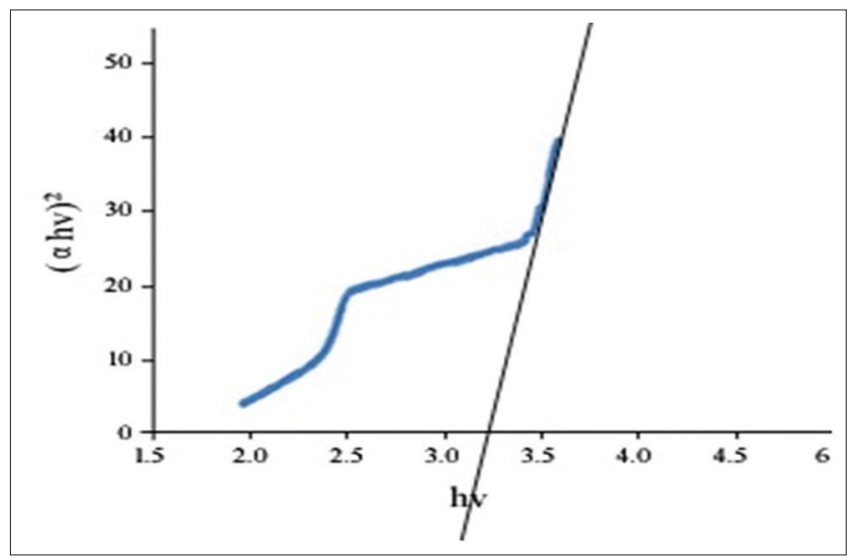

Fig. 26. Band gap energy for zinc oxide nanoparticles with benzalkonium chloride above critical micelle concentration.

\section{TABLE 1: Band gap energy for each sample}

\begin{tabular}{lcc}
\hline Status & $\begin{array}{c}\text { Band gap for } \\
\text { ZnO: CTAB }(\mathbf{e V})\end{array}$ & $\begin{array}{c}\text { Band gap for } \\
\text { ZnO: BAK }(\mathbf{e V})\end{array}$ \\
\hline Below CMC & 3.31 & 3.27 \\
At CMC & 3.49 & 3.24 \\
Above CMC & 3.39 & 3.21 \\
\hline
\end{tabular}

CTAB: Cetyltrimethylammonium bromide, ZnO: Zinc oxide, BAK: Benzalkonium chloride, CMC: Critical micelle concentration

\subsection{Analysis of X-Ray Diffractometer (X-Ray Diffraction [XRD])}

Fig. 27 shows the XRD patterns of the $\mathrm{ZnO}$ nanoparticles calcined at $450^{\circ} \mathrm{C}$ for $2 \mathrm{~h}$. A study of standard data JCPDS 760704 confirms that all the synthesized materials are hexagonal $\mathrm{ZnO}$ phase (wurtzite structure). The indexed spectrums of $\mathrm{ZnO}$ samples with CTAB at CMC, as shown in Fig. 28, have a high intensity and narrow width, which indicates that the products are well crystallized. 


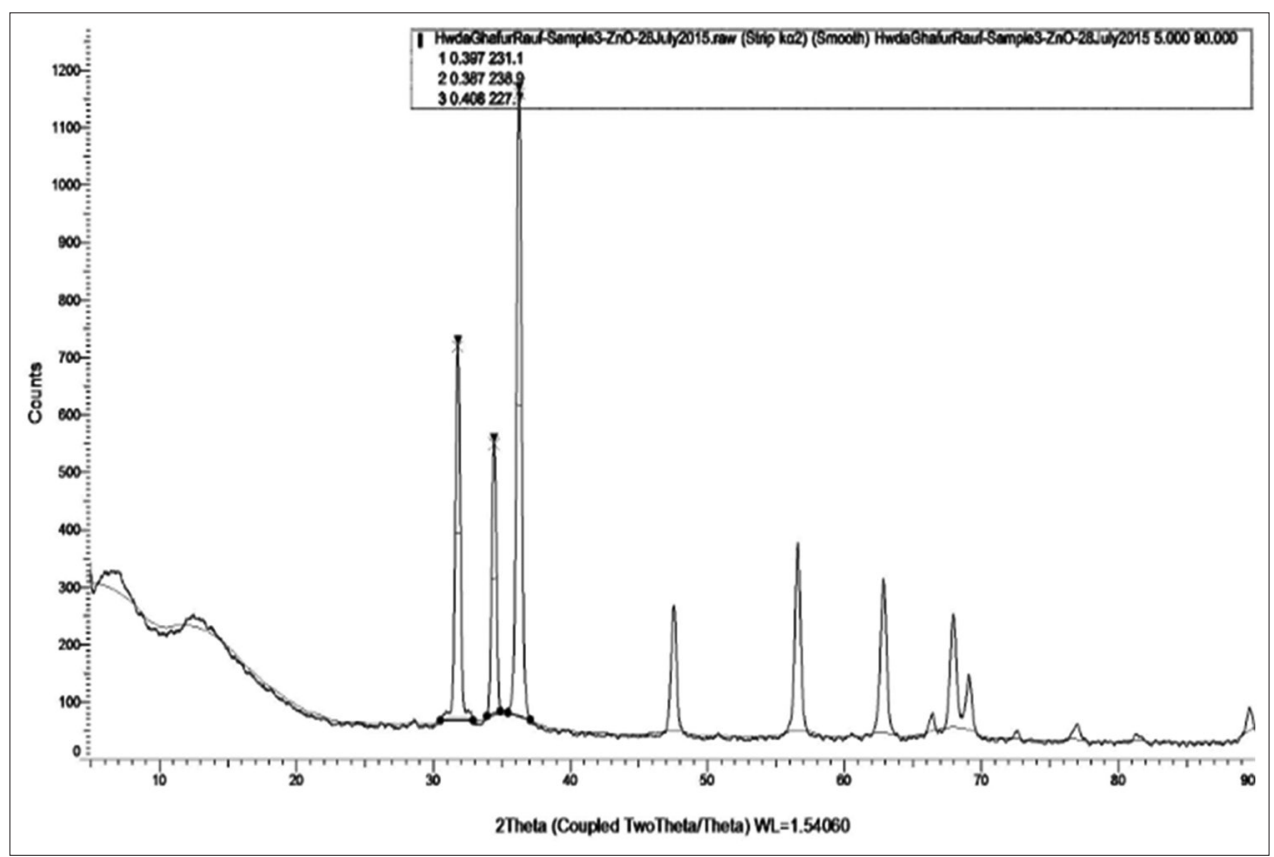

Fig. 27. XRD diffractogram of zinc oxide nanoparticles without surfactant.

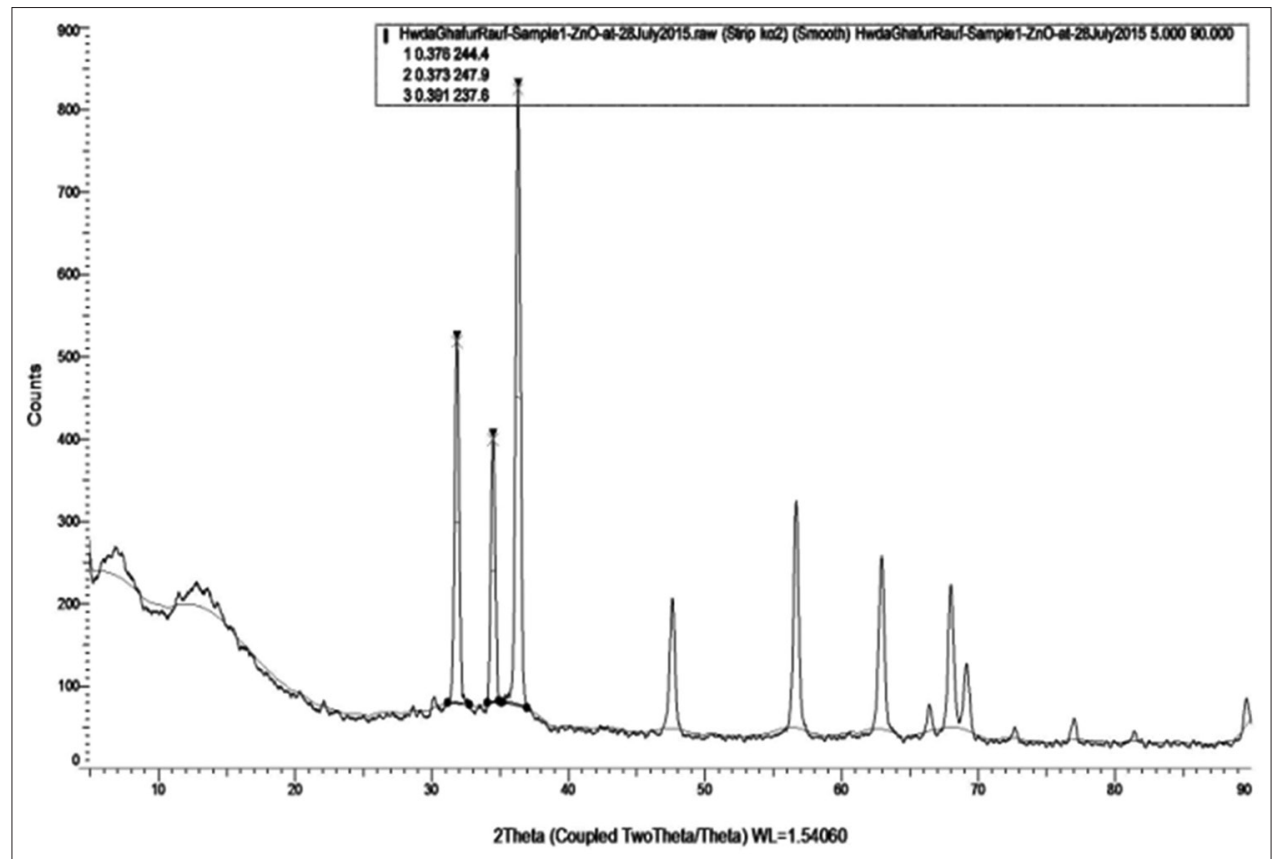

Fig. 28. XRD diffractogram of zinc oxide nanoparticles with cetyltrimethylammonium bromide at critical micelle concentration.

The XRD pattern for the $\mathrm{ZnO}$ nanoparticle prepared without surfactant calcined at $450^{\circ} \mathrm{C}$ for $2 \mathrm{~h}$ is shown in Fig. 27. The three highest spectrums were assigned as (100), (002), and (101) matched planes for $2 \Theta$ values of $31.769,34.422$, and $36.254^{\circ}$, respectively. The calculated average size of the most intense three diffraction spectrums was $76.9 \mathrm{~nm}$. However,
Fig. 29 is the $\mathrm{XRD}$ pattern for the $\mathrm{ZnO}$ nanoparticle prepared in the presence of CTAB below CMC calcined at $450^{\circ} \mathrm{C}$ for $2 \mathrm{~h}$, recorded similarity matching with $\mathrm{ZnO}$ nanoparticles prepared without surfactant based on reference (JCPDS 760704). To estimate the average crystallite sizes of the prepared $\mathrm{ZnO}$ nanoparticle, Debye-Scherrer equation was used for the 
(100), (002), and (101) planes corresponding to three exhibited broadened diffraction spectrums, and the average particle size was $74.1 \mathrm{~nm}$ (Fig. 29). This shows that the crystalline size becomes slightly smaller with increasing CTAB concentration.

The XRD pattern for the $\mathrm{ZnO}$ nanoparticle prepared in the presence of $\mathrm{CTAB}$ at $\mathrm{CMC}$ calcined at $450^{\circ} \mathrm{C}$ for $2 \mathrm{~h}$, as shown in Fig. 27, recorded the similarity matching with $\mathrm{ZnO}$ nanoparticles prepared without surfactant based on reference (JCPDS 76-0704) but there was a difference in the spectrum broadening, the diffraction spectrums became broader, which indicates the consistent of smaller particle sizes. They were assigned as (100), (002), and (101) for the three highest spectrums matched planes for $2 \Theta$ values of $31.769,34.422$, and $36.254^{\circ}$, respectively. The calculated average size of the most intense three diffraction spectrums was $68.0 \mathrm{~nm}$. This shows that the crystalline size became smaller in the presence of CTAB at $\mathrm{CMC}$ concentration compared to the one below $\mathrm{CMC}$. The reason is due to a large amount of surfactant monomers at CMC just before agglomeration and creating micelles. Moreover, the particles tend to agglomerate with the increase in CTAB surfactant concentration. Therefore, the amount of surfactant added was crucial in synthesizing $\mathrm{ZnO}$ with disperses particles. The surfactants create their own interface and form micelles at CMC [17]. Under the same conditions, micelle formation occurs in the bulk phase and surfactant-coated nanoparticles formed start to decrease by increasing surfactant concentration beyond the CMC point [18].
The XRD pattern of the $\mathrm{ZnO}$ nanoparticle prepared with $\mathrm{BAK}$ surfactant below $\mathrm{CMC}$ calcined at $550^{\circ} \mathrm{C}$ for $5 \mathrm{~h}$ is shown in Fig. 30. The three highest spectrums were assigned as (100), (002), and (101) matched planes for $2 \Theta$ values of 31.769, 34.422, and $36.254^{\circ}$, respectively. The structure of synthesized material is hexagonal $\mathrm{ZnO}$ (Wurtzite structure) according to standard data JCPDS 76-0704. The calculated average size of the most intense three diffraction spectrums was $116.4 \mathrm{~nm}$. Moreover, the structure was hexagonal as the others. On the other hand, Fig. 31 shows the XRD pattern of the $\mathrm{ZnO}$ nanoparticle prepared with $\mathrm{BAK}$ surfactant at $\mathrm{CMC}$ calcined at $550^{\circ} \mathrm{C}$ for $5 \mathrm{~h}$. The three highest spectrums were assigned as (100), (002), and (101) matched planes for $2 \Theta$ values of $31.769,34.422$, and $36.254^{\circ}$, respectively. The calculated average size of the most intense three diffraction spectrums was $130.0 \mathrm{~nm}$.

The XRD pattern for the $\mathrm{ZnO}$ nanoparticle prepared with BAK surfactant above $\mathrm{CMC}$ calcined at $550^{\circ} \mathrm{C}$ for $5 \mathrm{~h}$ is shown in Fig. 32. The three highest spectrums were assigned as (100), (002), and (101) matched planes for $2 \Theta$ values of $31.769,34.422$, and $36.254^{\circ}$, respectively. The calculated average size of the most intense three diffraction spectrums was $240.0 \mathrm{~nm}$. As its obvious the particle size of $\mathrm{ZnO}$ samples in the presence of BAK increased due to the bulky and bi-tailed structure of BAK which causes agglomeration. The tail of BAK contains 25 carbon atoms, which causes agglomeration and leads to increase in particle size [15].

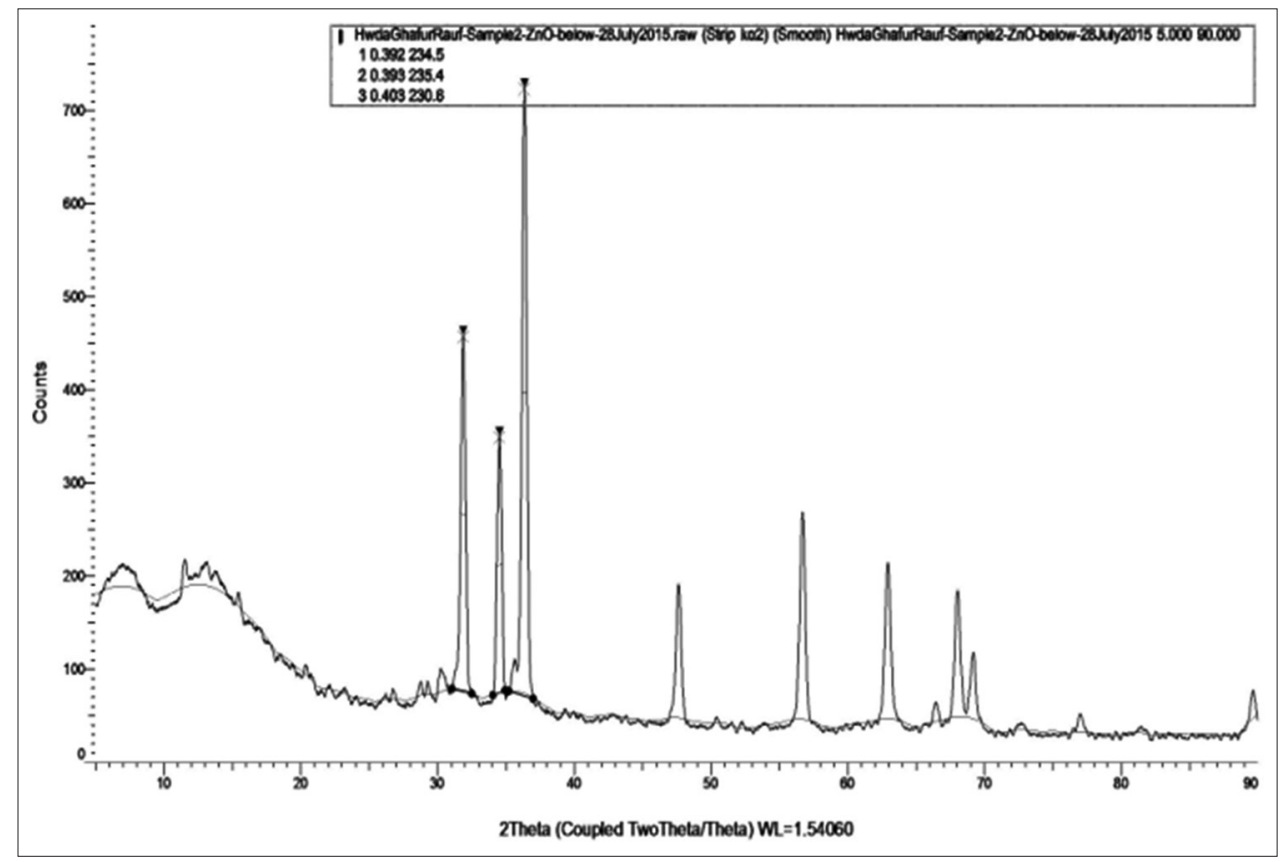

Fig. 29. XRD diffractogram of zinc oxide nanoparticles with cetyltrimethylammonium bromide below critical micelle concentration. 


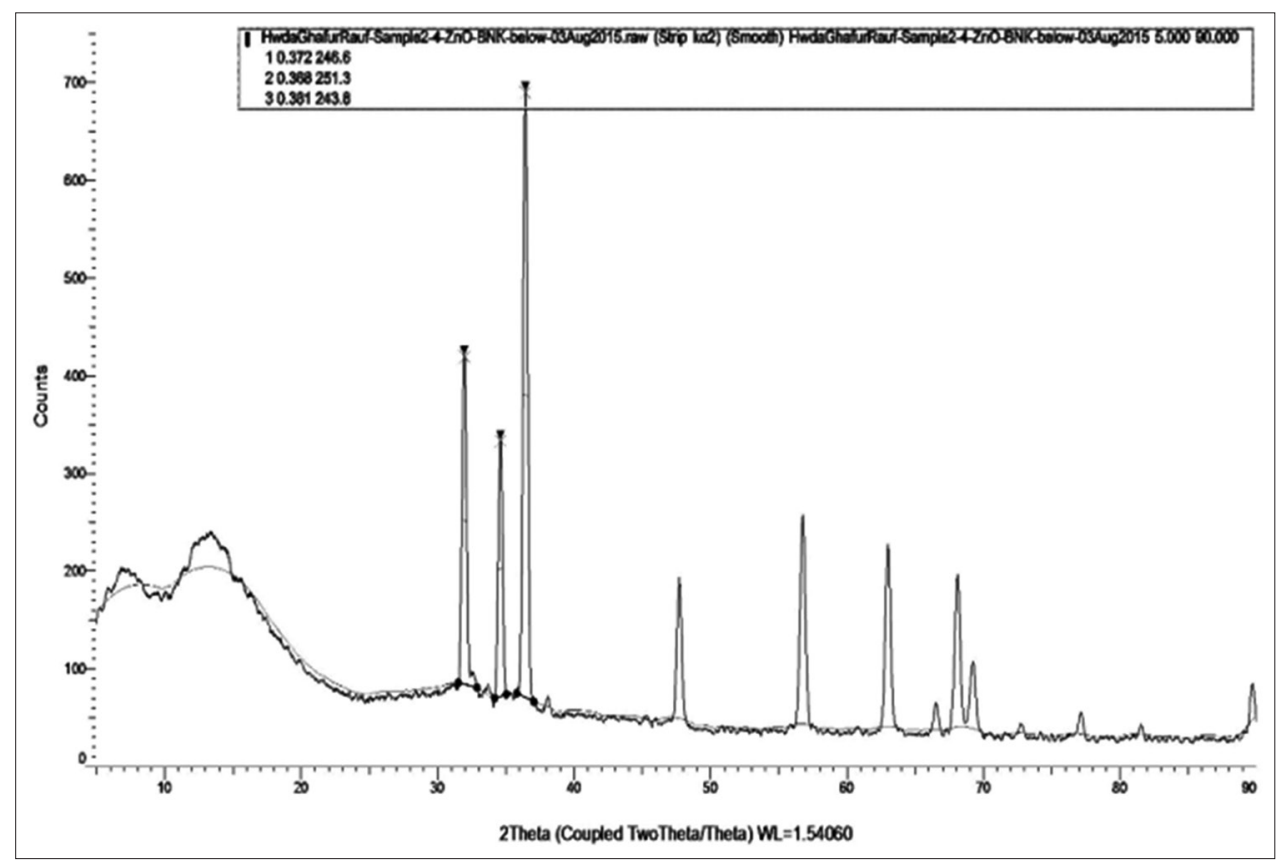

Fig. 30. XRD diffractogram of zinc oxide nanoparticles with benzalkonium chloride below critical micelle concentration.

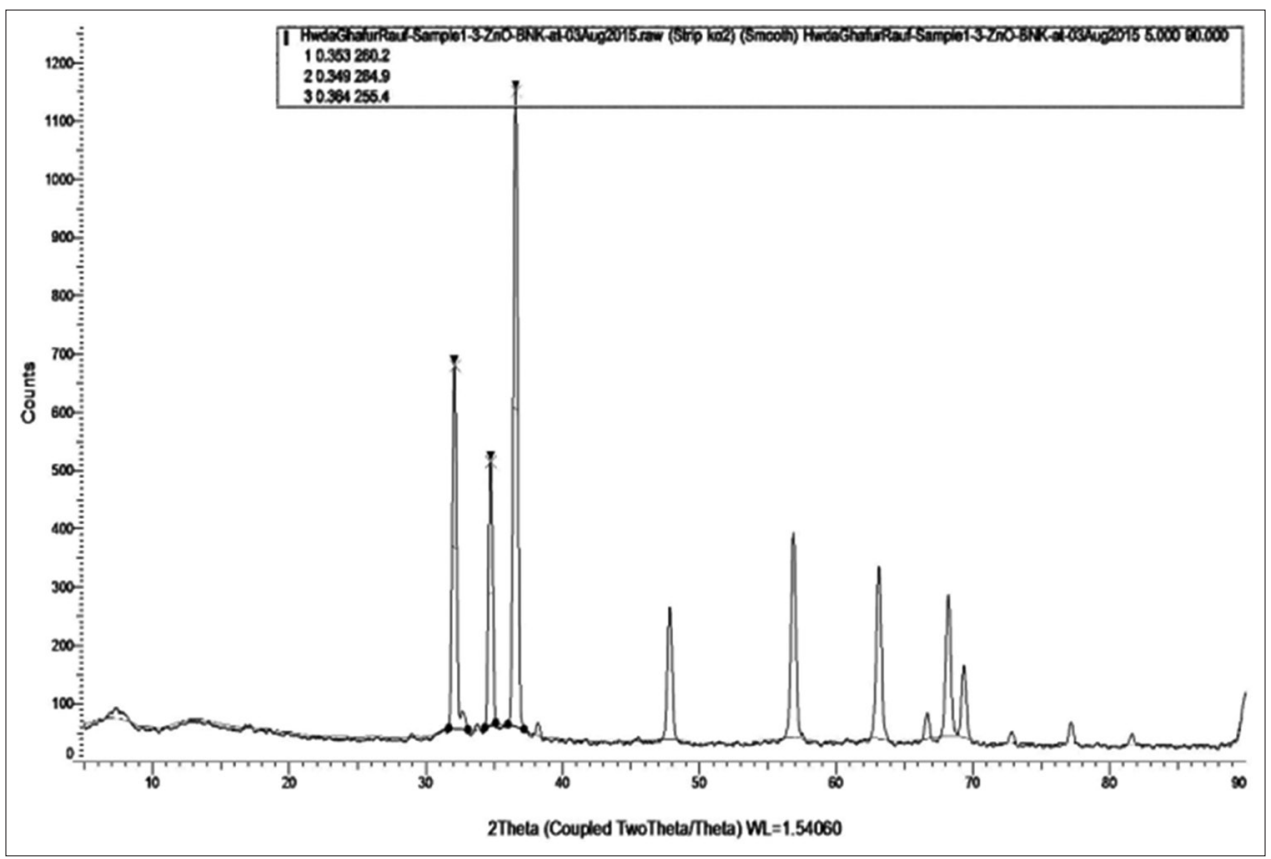

Fig. 31. XRD diffractogram of zinc oxide nanoparticles with benzalkonium chloride at critical micelle concentration.

\subsection{Analysis of Transmission Electron Microscopy (TEM)}

TEM was used to determine the average particle size by Digital Micrograph software. The results that obtained by TEM image and TEM particle size distribution of $\mathrm{ZnO}$ sample without surfactant were $66 \mathrm{~nm}$ and $60 \mathrm{~nm}$ for $\mathrm{ZnO}$ with $\mathrm{CTAB}$ below $\mathrm{CMC}, 51 \mathrm{~nm}$ for $\mathrm{ZnO}$ with CTAB at CMC, $100 \mathrm{~nm}$ for $\mathrm{ZnO}$ with BAK below CMC, and $140 \mathrm{~nm}$ for $\mathrm{ZnO}$ with $\mathrm{BAK}$ at $\mathrm{CMC}$, as shown in Figs. 33-42, respectively. As it is been mentioned in the previous investigations, the particle size of $\mathrm{ZnO}$ samples in the presence of BAK increased due to the bulky and 


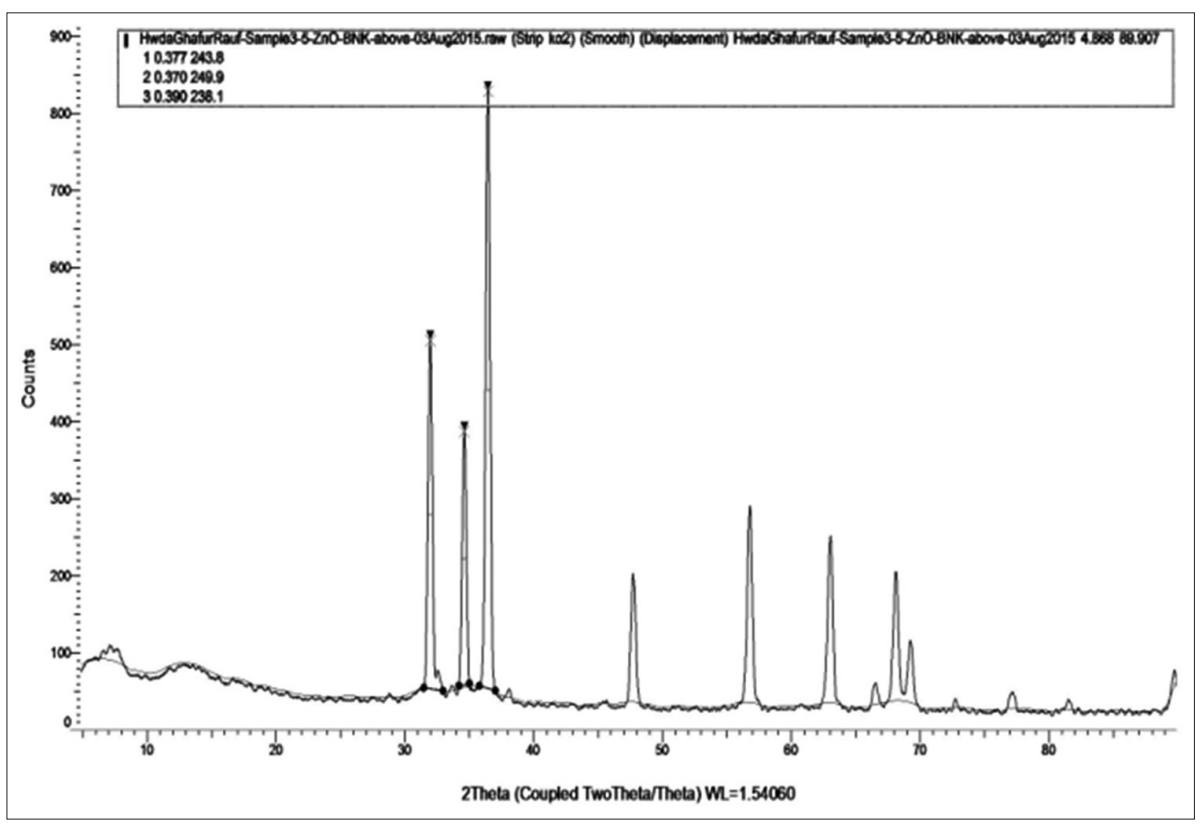

Fig. 32. XRD diffractogram of zinc oxide nanoparticles with benzalkonium chloride above critical micelle concentration.

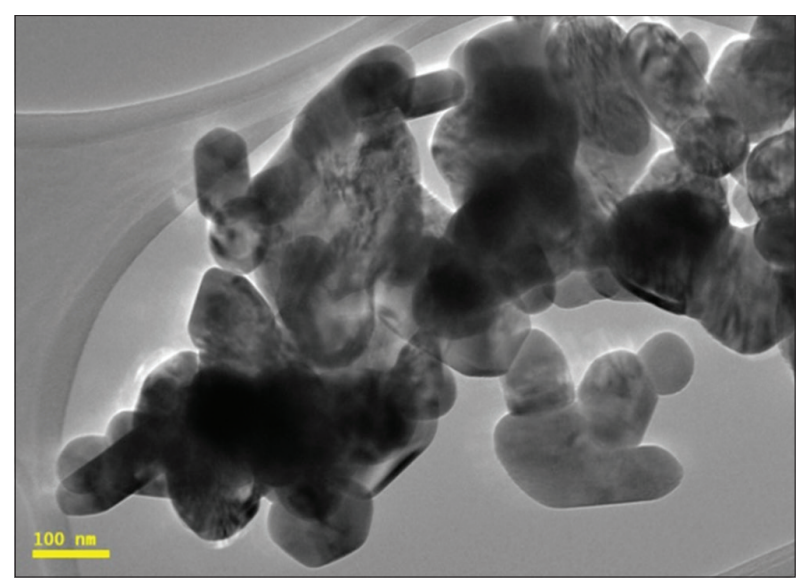

Fig. 33. Transmission electron microscopy image of zinc oxide nanoparticles without surfactant.

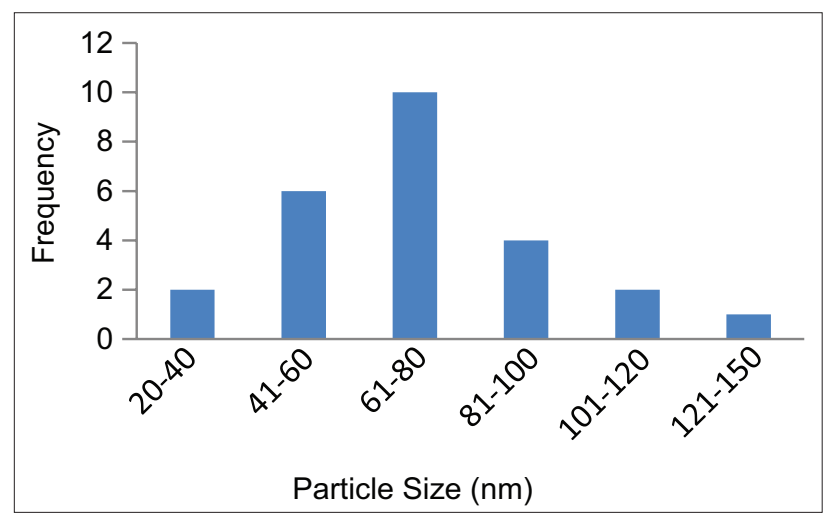

Fig. 34. Particle size distributions of zinc oxide without surfactant based on transmission electron microscopy.

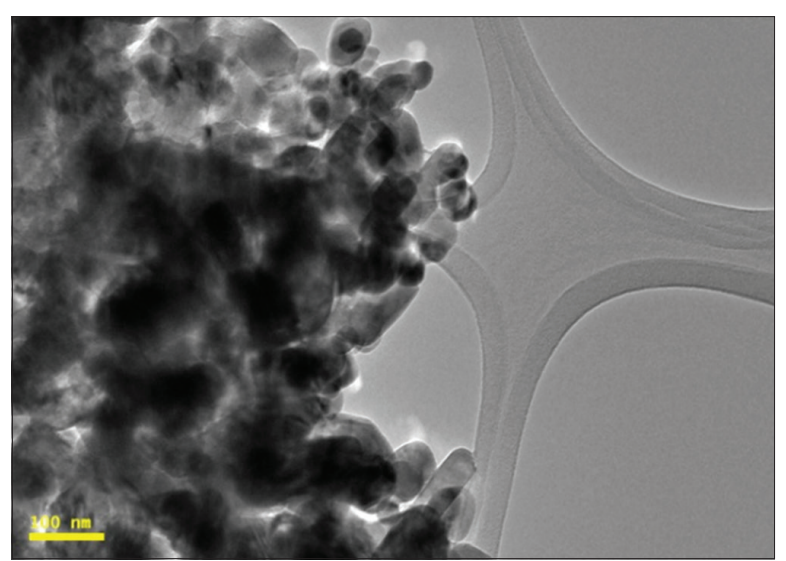

Fig. 35. Transmission electron microscopy image of zinc oxide nanoparticles with cetyltrimethylammonium bromide below critical micelle concentration.

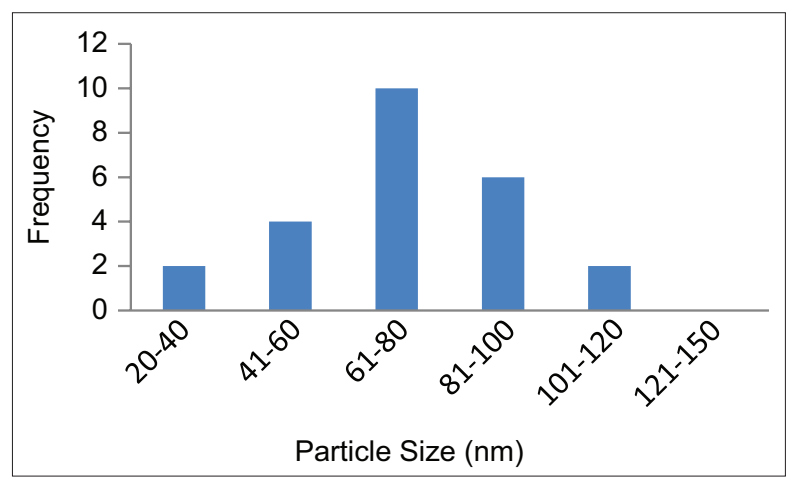

Fig. 36. Particle size distributions of zinc oxide with cetyltrimethylammonium bromide below critical micelle concentration based on transmission electron microscopy.

UHD Journal of Science and Technology | Jul 2019 | Vol 3 | Issue 2 


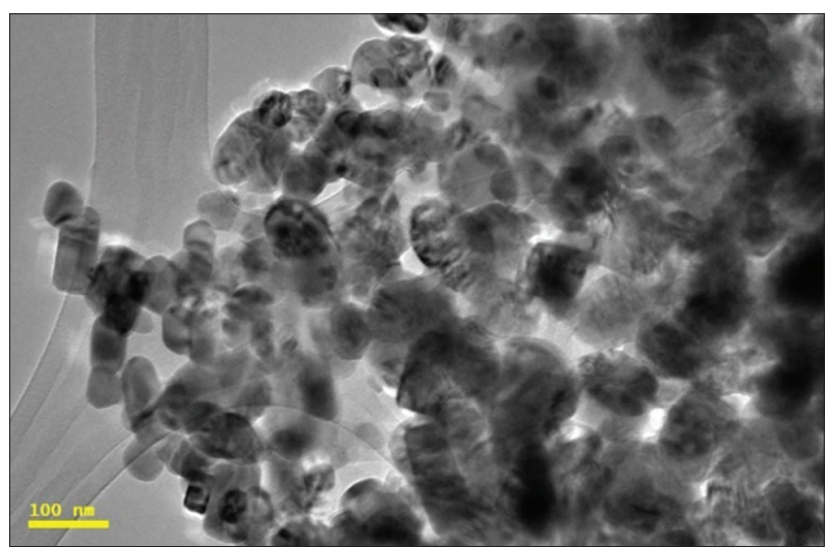

Fig. 37. Transmission electron microscopy image of zinc oxide nanoparticles with cetyltrimethylammonium bromide at critical micelle concentration.

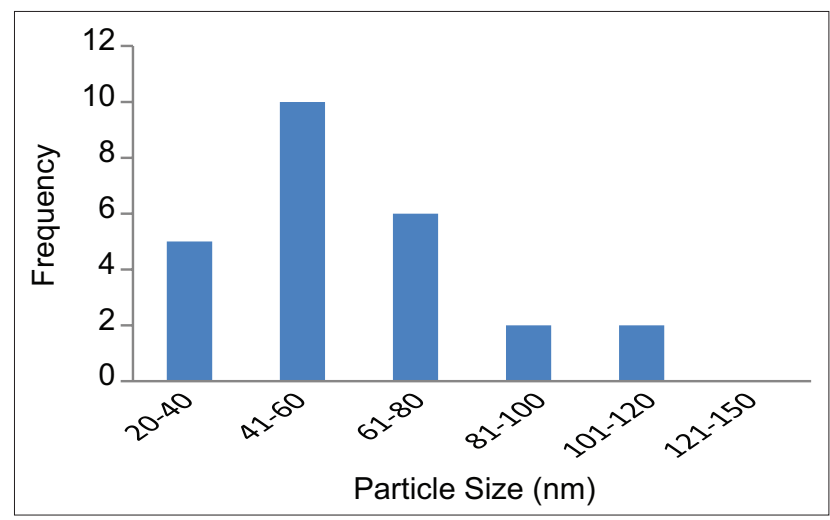

Fig. 38. Particle size distributions of zinc oxide with cetyltrimethylammonium bromide at critical micelle concentration.

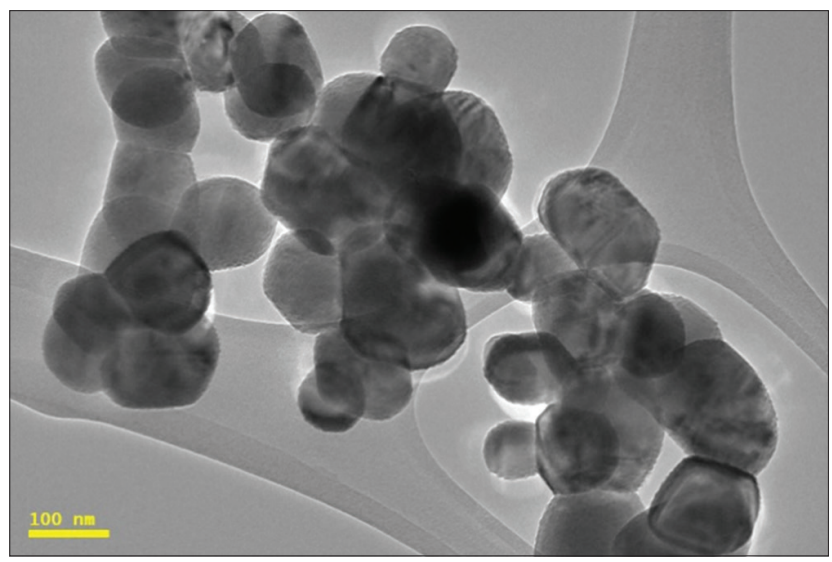

Fig. 39. Transmission electron microscopy image of zinc oxide nanoparticles with benzalkonium chloride below critical micelle concentration.

bi-tailed structure of BAK. The tail of BAK contains 25 carbon atoms, which causes agglomeration and lead to an increase in the particle size [15].

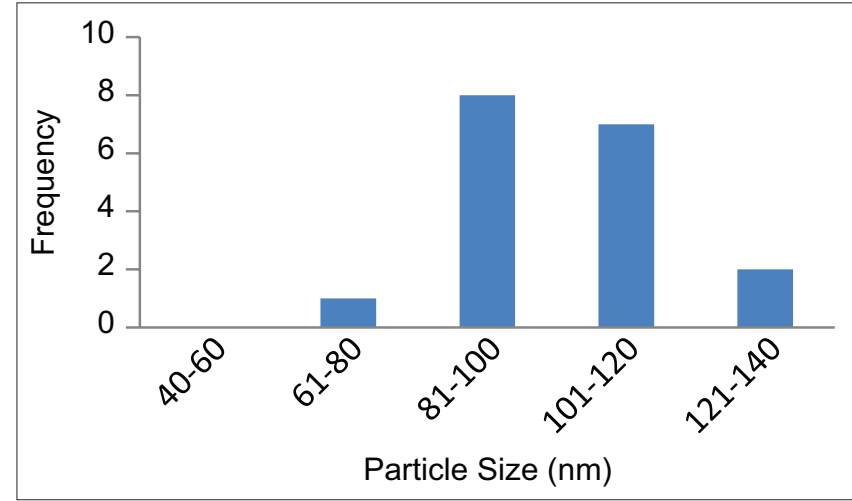

Fig. 40. Particle size distributions of zinc oxide with benzalkonium chloride below critical micelle concentration.

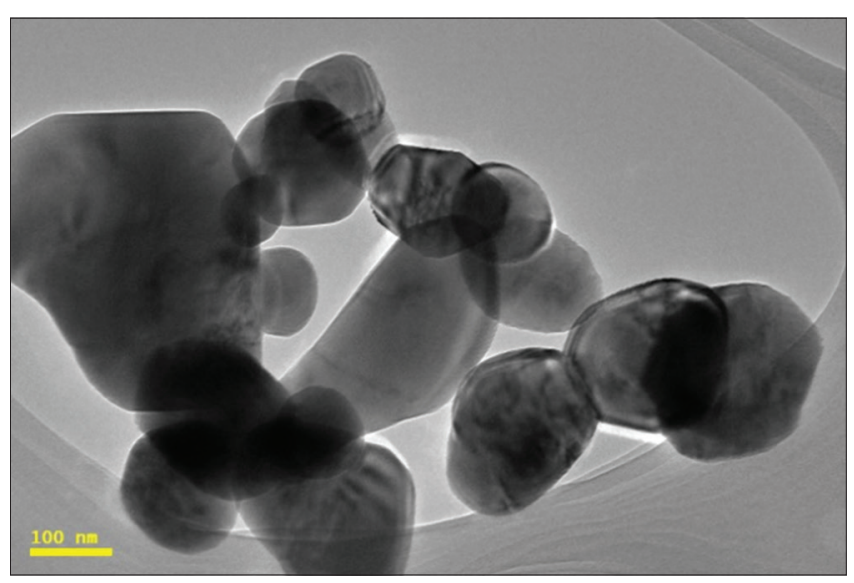

Fig. 41. Transmission electron microscopy image of zinc oxide nanoparticles with benzalkonium chloride at critical micelle concentration.

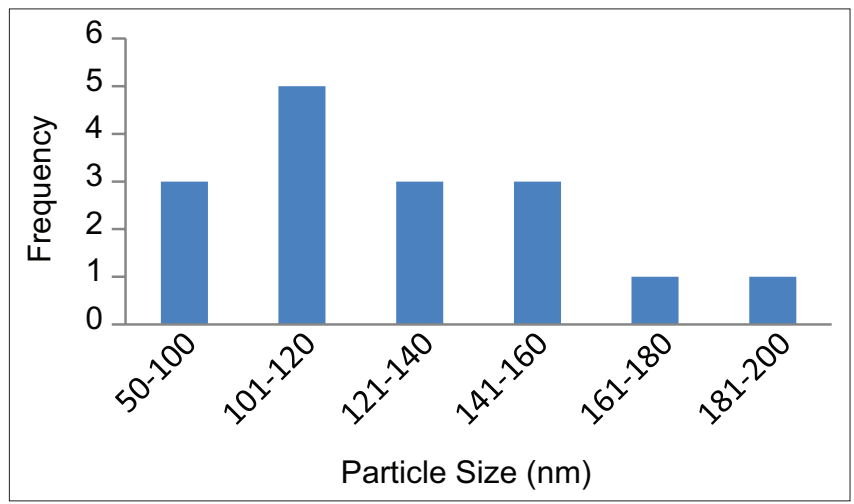

Fig. 42. Particle size distributions of zinc oxide with benzalkonium chloride at critical micelle concentration.

Among the samples, $\mathrm{ZnO}$ with $\mathrm{CTAB}$ at $\mathrm{CMC}$ has the smaller average particle size and starting to increase above $\mathrm{CMC}$ because the particles tend to agglomerate with the increase 
TABLE 2: Calculated particle sizes for each sample from XRD, TEM, and SEM in different conditions

\begin{tabular}{lcccc}
\hline Status & Temperature $\left({ }^{\circ} \mathbf{C}\right)$ & $\begin{array}{c}\text { Average particle size } \\
\text { from XRD }(\mathbf{n m})\end{array}$ & $\begin{array}{c}\text { Average particle size } \\
\text { from TEM }(\mathbf{n m})\end{array}$ & $\begin{array}{c}\text { Average particle size } \\
\text { from SEM }(\mathbf{n m})\end{array}$ \\
\hline ZnO without surfactant & 500 & 76.9 & 66 & 87 \\
ZnO with CTAB below CMC & 500 & 74.1 & 60 & 84 \\
ZnO with CTAB at CMC & 500 & 68 & - & 73 \\
ZnO with CTAB above CMC & 500 & - & 100 & 140 \\
ZnO with BAK below CMC & 500 & 116.4 & 140 & 125 \\
ZnO with BAK at CMC & 500 & 130 & - & 212 \\
ZnO with BAK above CMC & 500 & 240 & & 212 \\
\hline
\end{tabular}

ZnO: Zinc oxide, CTAB: Cetyltrimethylammonium bromide, BAK: Benzalkonium chloride, SEM: Scanning electron microscopy, TEM: Transmission electron microscopy, CMC: Critical micelle concentration

in CTAB surfactant concentration. The surfactants create their own interface and form micelles at CMC. Therefore, the amount of surfactant added was crucial in synthesizing $\mathrm{ZnO}$ with disperses particles.

The calculated particle sizes for each sample from XRD, TEM, and SEM are depicted in Table 2.

\section{CONCLUSION}

Among the prepared samples in the presence of CTAB at $\mathrm{CMC}$, the prepared $\mathrm{ZnO}$ nanoparticles show uniform morphology, demonstrate a smaller size compared to the others, and the size increases with increasing CTAB concentration (after $\mathrm{CMC}$ ), as the particles tend to agglomerate by increasing CTAB surfactant concentration. Moreover, with the addition of BAK surfactant, the particle size of all the samples starts to increase due to the carbon number in the structure of BAK which is for both tails is 25 , which means surfactant activity becomes minimal and insoluble. Thus, solubility and practical surfactant properties are somewhat related. While the number of carbon atoms in the tail for CTAB is 17 at such level, a surfactant has good but limited solubility in water [15].

If the concentration of the surfactant was larger than the $\mathrm{CMC}$, the surfactant formed micelles. When micelles are fully formed, the latter is not effective in dispersing the particles compared to monomers, which leads to an increase in particle size [19]. Agglomerations of particles occur in the micelle formation of surfactant, which results in larger particle size when the ratio of surfactant increases [19]. The indexed spectrums of $\mathrm{ZnO}$ with CTAB at CMC in the XRD spectrums have a high intensity and narrow width, which indicates that the products are well crystallized. Large band gap energy and highly blue-shifted absorption edge confirm that the synthesized $\mathrm{ZnO}$ nanoparticles in the presence of $\mathrm{CTAB}$ at $\mathrm{CMC}$ exhibit a strong quantum confinement effect [20].

\section{ACKNOWLEDGMENTS}

The authors are thankful to Prof. Madzlan University Technology Malaysia for his review and guidance.

\section{AUTHORS' CONTRIBUTIONS}

Hwda Rauf was responsible for data collection and analysis and for preparing the manuscript draft. Hamid Rasheedi worked on the concept of the given study and on the final manuscript review. Both authors read and approved the final manuscript.

\section{REFERENCES}

[1] E. A. Meulenkamp. "Synthesis and growth of $\mathrm{ZnO}$ nanoparticles." The Journal of Physical Chemistry B, vol. 102, no. 29, pp. 55665572, 1998.

[2] D. Li and R. B. Kaner. "Shape and aggregation control of nanoparticles: Not shaken, not stirred". Journal of the American Chemical Society, vol. 128, no. 3, pp. 968-975, 2006.

[3] V. Nandwana, K. E. Elkins, N. Poudyal, G. S. Chaubey, K. Yano and J. P. Liu. "Size and shape control of monodisperse FePt nanoparticles". The Journal of Physical Chemistry C, vol. 111, no. 11, pp. 4185-4189, 2007.

[4] T. Nasiru, L. Avila and M. Levine. "Determination of critical micelle concentration using UV visible spectroscopy". Journal of High School Research, vol. 2, pp. 1-5, 2011.

[5] Y. Shimizu, A. Jono, T. Hyodo and M. Egashira. "Preparation of large mesoporous SnO2powder for gas sensor application". Sensors and Actuators B: Chemical, vol. 108, no. 1, pp. 56-61, 2005.

[6] C. Vautier-Giongo and B. L. Bales. "Estimate of the ionization degree of ionic micelles based on Krafft temperature measurements". The Journal of Physical Chemistry B, vol. 107, no. 23, pp. 5398-5403, 2003.

[7] Sccnfp. "Opinion of the Scientific Committee on Cosmetic Products 
and Non-food Products Intended for Consumers, 2003. Available from: http://www.ec.europa.eu/health/archive/ph_risk/committees/ sccp/documents/out250_en.pdf. [Last accessed on 2019 Sep].

[8] M. Gusatti, J. A. Rosário, G. S. Barroso, C. E. M. Campos, H. G. Riella and N. C. Kunhen. "Synthesis of $\mathrm{ZnO}$ nanostructures in low reaction temperature". Chemical Engineering Transactions, vol. 17, pp. 1017-1021, 2009.

[9] D. J. Sornalatha and P. Murugakoothan. "Room temperature synthesis of $\mathrm{ZnO}$ nanostructures using CTAB assisted solgel method for application in solar cells". Journal of Emerging Technology and Advanced Engineering, vol. 3, no. 9, pp. 414-418, 2013.

[10] S. P. Ghosh. "Synthesis and Characterization of Zinc Oxide Nanoparticles by Sol-Gel Process". National Institute of Technology, Rourkela, 2012

[11] J. Eastoe and D. Sharpe. "Properties of phosphocholine microemulsions and the film rigidity model". Langmuir, vol. 13, no. 13, pp. 3289-3294, 1997.

[12] P. C. Joanna, E. M. Michael and B. T. Jason. "Solvent effects on copper nanoparticle growth behavior in AOT reverse micelle system". The Journal of Physical Chemistry B, vol. 105, pp. 22972302, 2001.

[13] Y. Gargouri, R. Julien, A. G. Bois, R. Verger and L. Sarda L. "Studies on the detergent inhibition of pancreatic lipase activity". Journal of Lipid Research, Vol. 24, no. 10, pp. 1336-1342, 1983.

[14] K. Anandan and V. Rajendran. "Structural, optical and magnetic properties of well-dispersed $\mathrm{NiO}$ nanoparticles synthesized by CTAB assisted solvothermal process". Nanoscience. Nanotechnology International Journal, vol. 2, no. 4, pp. 24-29, 2012.

[15] R. Mandavi, S. K. Sar and N. Rathore. "Critical micelle concentration of surfactant, mixed surfactant and polymer by different method at room temperature and its importance". Oriental Journal of Chemistry, vol. 24, no. 2, pp. 559-564, 2008.

[16] C. Fetzer, R. T. Lee and G. B. Stringfellow. "Effect of surfactant Sb on carrier lifetime in GalnP epilayers". Journal of Applied Physics, vol. 91, no. 1, pp. 199-203, 2002.

[17] M. A. Farrukh, P. Tan and R. Adnan. "Influence of reaction parameters on the synthesis of surfactant-assisted tin oxide nanoparticles". Turkish Journal of Chemistry, vol. 36, no. 2, pp. 303-314, 2012.

[18] E. Y. Bryleva, N. Vodolazkaya, N. Mchedlov-Petrossyan, L. Samokhina, N. Matveevskaya and A. Tolmachev. "Interfacial properties of cetyltrimethylammonium-coated $\mathrm{SiO} 2$ nanoparticles in aqueous media as studied by using different indicator dyes". Journal of Colloid and Interface Science, vol. 316, no. 2, pp. 712722, 2007.

[19] O. A. Graeve, H. Fathi, J. P. Kelly, M. S. Saterlie, K. Sinha, G. Rojas-George, R. Kanakala, D. R. Brown and E. A. Lopez. "Reverse micelle synthesis of oxide nanopowders: Mechanisms of precipitate formation and agglomeration effects". Journal of Colloid and Interface Science, vol. 407, pp. 302-309, 2013.

[20] M. S. Samuel, L. Bose and K. George. "Optical properties of ZnO nanoparticles". Academic Review, vol. 16, pp. 57-65, 2009. 